Research Article

\title{
Modeling of Linear Displacement and Design of Adaptive Robust Controller
}

\author{
Lei Jiang \\ Information Engineering Department, China University of Mining and Technology-Beijing, Beijing 100083, China \\ Correspondence should be addressed to Lei Jiang; leijiang@cumtb.edu.cn
}

Received 30 May 2020; Revised 20 October 2020; Accepted 30 October 2020; Published 16 November 2020

Academic Editor: Vincenzo Vespri

Copyright (c) 2020 Lei Jiang. This is an open access article distributed under the Creative Commons Attribution License, which permits unrestricted use, distribution, and reproduction in any medium, provided the original work is properly cited.

Twisted-string actuation method has attracted much more attention due to its compact size and considerable output force. Many studies have been proposed to estimate its linear displacement of actuation based on the angular measurement of the rotary device. However, the estimated results are not always accurate, which requires an additional process to make the estimation accuracy. In this paper, an ARC control scheme with force feedback has been developed for a high-accuracy twisted-string actuation system.

\section{Introduction}

As robotic applications have started to gain much more attention recently, it is desired to have actuators that can generate large torque while keeping its size tiny. However, with the addition of the required power source, most available solutions of actuators are still bulky. These actuators might not be too heavy for the applications of wearable robotic devices. Among all different actuators, twisted-string actuation has the advantages of lightweight by moving bulky components to other locations while providing enough force or torque to drive moving parts. It has attracted many research groups to adopt this twisted-string actuator in various exoskeleton robotic applications [1-4].

A twisted-string actuation system comprises of two or more parallel strands that convert rotational movement to linear displacement. The configuration is shown in Figure 1. Once the string starts to be twisted, the length is shortened. With carefully selected material and an accurate model, such an actuation method can serve as an actuator that delivers linear movement. It also has the advantage of keeping the rotary driving component, which is typically bulky, at a different location to keep the weight at the location of output torque, or force, being light. Such actuators have attracted much attention since Suzuki et al. adopted it to control the stiffness of the links in the application of robotic hands [5].
Würtz et al. have presented a simple kinetostatic model of a twisted-string-based transmission system [6].

However, the simple model does not describe specific nonlinear phenomena in the physical equations. Thus, more complex models have been proposed to better approximate the contraction and extension for the twisted-string actuation $[7,8]$. These models discuss more on the string with dual-strand, which might limit the applicable range of such an actuation device. Shisheie et al. have investigated the performance of various combinations of strands [9]. Their result has illustrated that the trajectories of hysteresis are different when the number of integrated strands is changed. A string with more strands can yield larger friction. However, the model has still not been investigated yet. A model that can accurately estimate the actuated length of both contraction and expansion is particularly important since the changed length typically cannot be measured directly. Available sensors can only measure other physical properties and estimate the real length change indirectly. Though the modified model proposed by Gaponov et al. [8], it does not perfectly estimate the internal reaction among strands. It is also challenging to determine the output force of such kind of actuator. In this study, a nonlinear approach is proposed to enhance the accuracy of the estimation of length estimation and estimate the output force of twisted-string actuation. 


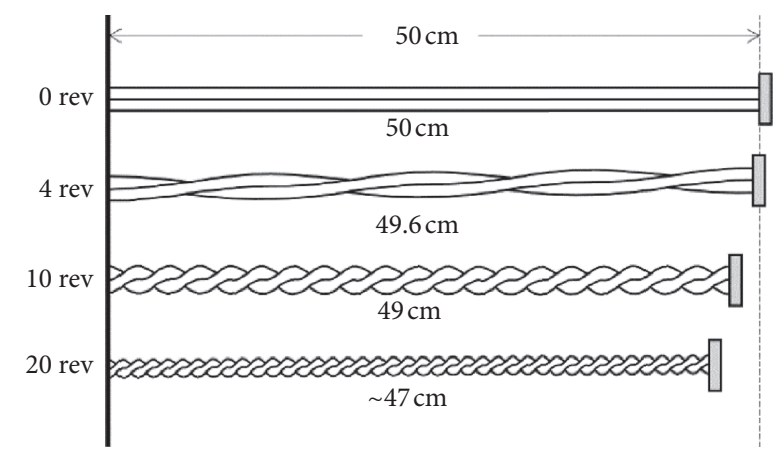

FIGURE 1: Length variation of twisted actuation after 4,10 , and 20 revolutions.

Instead of using an expensive optical linear encoder, this study adopted an adaptive robust control algorithm with the force feedback to compensate for nonlinear factors of the twisted-string actuation.

\section{Experimental Setup}

To validate different mathematical models and the corresponding reliabilities, an experimental platform was built to characterize the relationship between the revolutions of the driving motor and the amount of contraction or expansion of the twisted-string using different string types and configurations.

To ensure the contraction and expansion to be linear movements, two $400 \mathrm{~mm}$ Richelieu slides were used to guide the movements. Figure 2 illustrates the schematic configuration of the experimental setup. In this setup, a DC motor was placed on the upper side of the testbed with a string holder attached to the shaft. The string holder has 7 holes with one hole located at the center and 6 holes evenly on a circular path [9]. Depending on the types of tested string, as shown in Figure 3, different types of strings with the required number of strands were used in individual tests. The same holder was fixed on a slider, which transforms the rotational motion into linear movement.

The original length of a single strand is designed to be $500 \mathrm{~mm}$ or $1,000 \mathrm{~mm}$. The DC motor, a high-torque Pololu motor with a 19:1 gear ratio and a 64 CPR encoder, used to twist the string is driven by a PWM mode L298 H-Bridge. In addition to the rotary encoder, several sensors were equipped as well. A WXY linear encoder is used to measure the linear displacements of the twisted-string; the resolution of linear encoder is $0.04 \mathrm{~mm}$. A high precision sliding potentiometer was also used to detect the linear displacement. An AC712 current sensor is used to measure the current of the armature of the DC motor. A SHIMPO FGVXY20 is used to measure the tensile force generated by the twisted-string, the resolution of the force gauge $0.1 \mathrm{~N}$. To acquire the movement and force data, a desktop PC with a Pentium IV $2.4 \mathrm{GHz}$ and $1 \mathrm{~GB}$ memory is used for data acquisition. Two data acquisition I/O cards (PCI-6221 and PCI-6601) manufactured by the National Instrument is used to generate command signals to motor drivers and to acquire signals from position sensors, current sensor, and

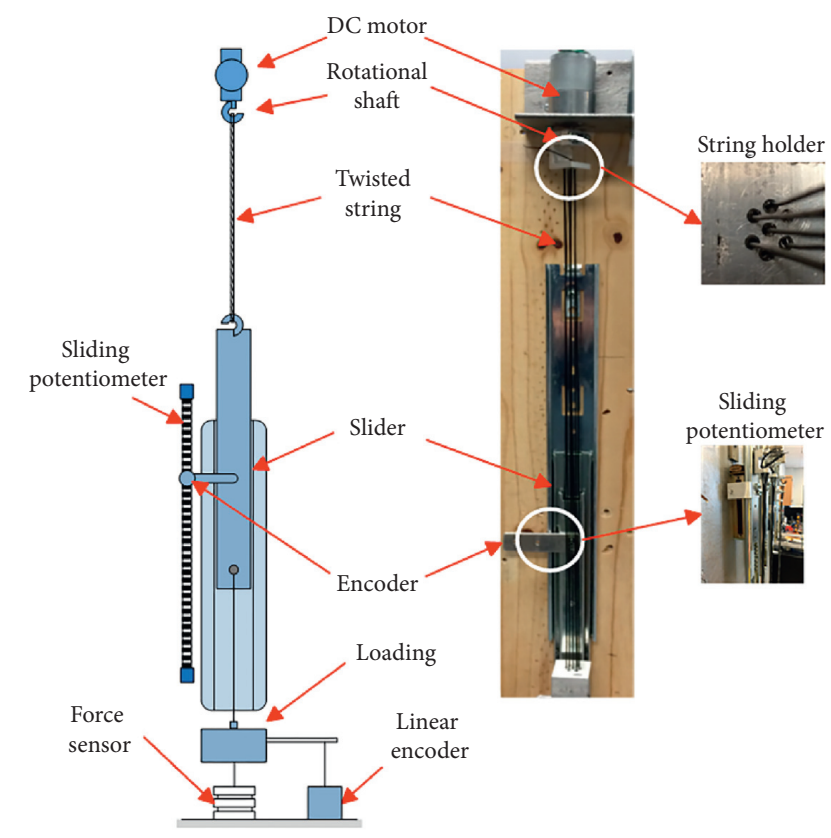

FIGURE 2: Schematic diagram of the experimental platform and the physical setup.

force sensor. The data acquisition program was developed using MATLAB Simulink with xPC Toolbox. The sampling rate is $100 \mathrm{~Hz}$.

To guarantee smooth movement during each test, a mass was added to the lower end of the twisted-string. The friction of the guided movement in both moving directions was investigated ahead. With the proposed experimental setup, the friction of upward motion is around $0.52 \mathrm{~N}$, and it is around $0.14 \mathrm{~N}$ for the downward movement.

\section{Models of Twisted-String Actuation}

The basic concept of the twisting actuation method is simple. With one end fixed on an actuator shaft (electric motor) and the other end fixed on the load, two or more parallel strands can be twisted by the rotational movement of the attached actuator. As the twist starts, the string connected to the motor shaft starts to reduce the distance between both ends and generates linear motion.

A mathematical model for such a transmission mechanism has been proposed by Würtz et al. [6]. This model assumes the stiffness of adopted strands being infinite, which can only be correct if the exerted force on the strands is small relative to the elastic modulus of the string. There are eight configurations of strand combinations used by twistedstring actuation, distinguished by different strands of actuation and with/without a neutral strand, as shown in Figure 3. One configuration includes a neutral strand, which increases the effective radius about as the surrounding strands wrap the neutral one. The other configuration does not include a neutral strand. The distance $r$ between the effective strands and center of rotation becomes $r=r_{s}+r_{c}$, where $r_{s}$ is the radius of the central neutral strand, and $r_{c}$ is the radius of the effective strands. 

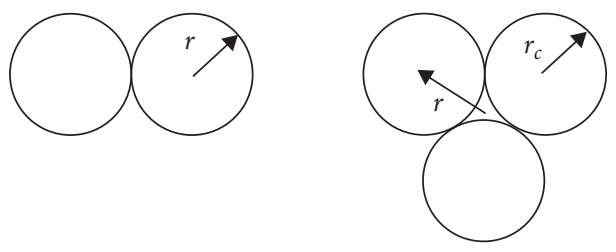

(b)

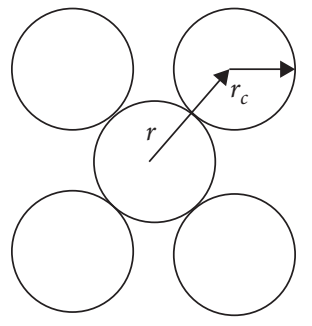

(e)

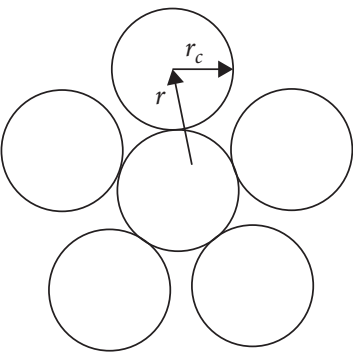

(f)
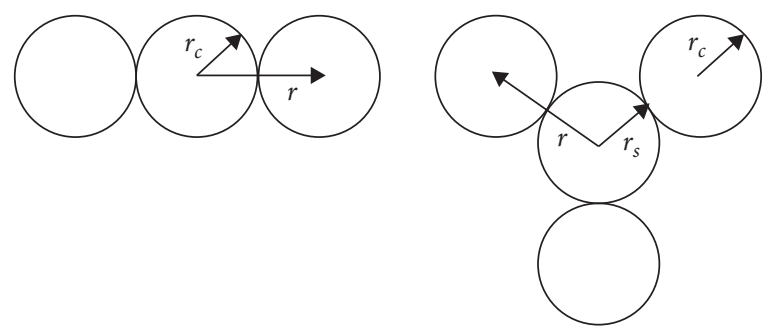

(d)

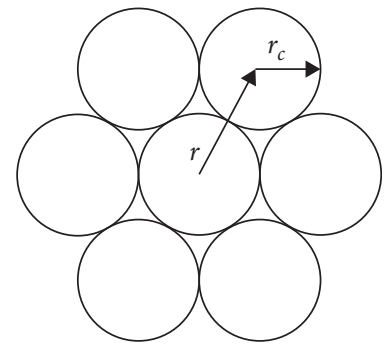

(g)

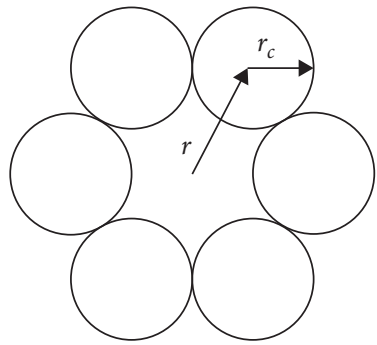

(h)

Figure 3: Different configurations of the strings used in the experiment: twisted-string configuration of a 2-strand actuation (a) and (c) and 3-strand actuation (b) and (d) with and without a neutral strand.

The kinematic relationship between the motor rotation $\theta$ and the length of the transmission system $p$ is derived according to Pythagoras' theorem, which is shown in Figure 4 . The actuation length $p$ is a function of the number of rotations of the actuator:

$$
p=\sqrt{L^{2}-\theta^{2} r^{2}}
$$

where $L$ is the total length of the string. Starting from completely untwisted-strings, the change in the actuation length $\Delta p$ will be

$$
\Delta p=L-\sqrt{L^{2}-\theta^{2} r^{2}} .
$$

To maintain the static equilibrium at different positions, sufficient torque $\tau_{m}$ must be generated by the actuator. In other words, to stay at equilibrium, the static compound force $\left(n F_{i}\right)$ generated by $F_{z}$ as the tangential force (load), and $F_{\tau}$ as the axial force corresponding to $\tau_{m}$ must be aligned with the physical direction of the unwrapped helix. To provide dynamic actuation, the provided torque should be more or less than the required static torque.

The amount of actuator force depends directly on the amount of load and helix slope of the strand, which is

$$
\begin{aligned}
F_{i} & =\frac{F_{z}}{n \cos (\alpha)}, \\
F_{\tau} & =n F_{i} \sin (\alpha), \\
\tau_{m} & =r F_{\tau},
\end{aligned}
$$

where $n$ and $\alpha$ are the number of strands and helix slope, respectively. By combining equations of (3), the torque $\tau_{m}$ becomes

$$
\tau_{m}=r F_{z} \tan (\alpha)=F_{z} \theta \frac{r^{2}}{p} .
$$

In cases of high loads or accelerated motion, the assumption of infinite stiffness of the string is no longer valid and the elongation of the strands due to the axial force must be taken into account. In this case, the total length of a string $L$ is a function of the tension $F_{i}$, the strand stiffness $k$, and its unloaded length $L_{0}$. Thus, (1) can then be modified to

$$
p=\sqrt{L_{0}^{2}\left(1+\frac{F_{i}}{L_{0} k}\right)^{2}-\theta^{2} r^{2}}
$$

With (5), the equation to determine the length of pretwist actuation can be explained as

$$
p_{\theta_{0}}=\sqrt{L_{0}^{2}\left(1+\frac{F_{i}}{L_{0} k}\right)^{2}-\theta_{0}^{2} r^{2}}
$$

where $\theta_{0}$ is the number of revolutions of the actuator associated with the pretwist. Assuming that this is the initial length of the cable, $p(\theta)$ as a function of the revolution of the motor can be derived as follows:

$$
P\left(\theta, F_{i}\right)=\sqrt{L_{0}^{2}\left(1+\frac{F_{i}}{L_{0} k}\right)^{2}-\theta^{2} r^{2}}-\sqrt{L_{0}^{2}\left(1+\frac{F_{i, \theta}}{L_{0} k}\right)^{2}-\theta_{0}^{2} r^{2}},
$$

where $P\left(\theta, F_{i}\right)$ is the amount of displacement from the pretwist position. According to the geometric relationship, there exists a maximum angle $\alpha$ where all strands are wrapped tightly on each other. After this point, strands begin entangling around themselves. With the maximum angle $\alpha$, the pitch parameter $q$ of the helix is minimized:

$$
q_{\min }=2 n r_{s} \text {. }
$$




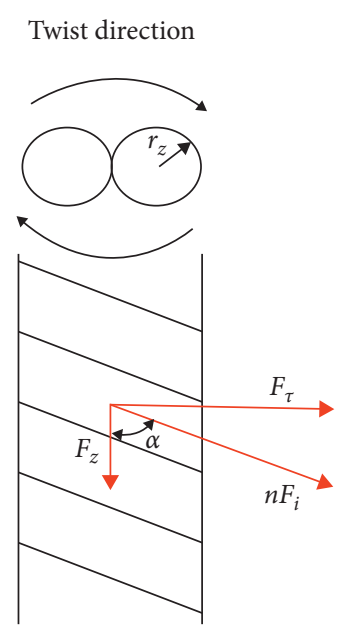

(a)

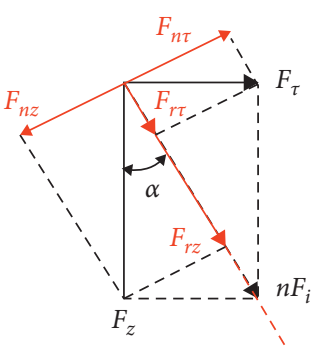

(b)

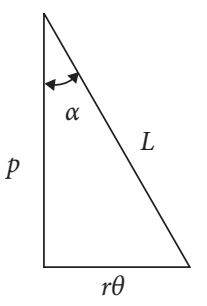

(c)

Figure 4: (a) Schematic representation of twisted-string and forces; (b) equilibrium of acting forces; (c) unwrapped helix.

On the other hand, the pitch parameter of a helix as a function of motor rotation angle $\theta$ and string length $p$ will be

$$
2 \pi p=q \theta .
$$

Assuming no load $\left(F_{z}=0\right)$ was applied to the transmission and combining (5) with the geometry of the strands, (8) and (9), the maximum achievable $\theta$ angle $\left(\theta_{\max }\right)$ and minimum contraction $\left(p_{\min }\right)$ are, respectively,

$$
\begin{aligned}
& \theta_{\max }=\frac{L_{0}}{\sqrt{r^{2}+\left(r_{s}^{2} n^{2} / \pi^{2}\right)}}, \\
& p_{\min }=\frac{L_{0}}{\sqrt{1+\left(r^{2} \pi^{2} / r_{s}^{2} n^{2}\right)}} .
\end{aligned}
$$

\section{Verification of Theoretical Estimation}

To verify the correctness of the theoretical estimation and select the optimal configuration and material of the strands for the twisted-string actuation which requires low hysteresis, quick response, and better durability, three types of material that were tested: a monofilament fishing line, a utility cable, and a tactical cable. In these tests, the behavior of the actuation within the low-hysteresis area was investigated. Since the actuations were twisted to the maximum limit and in some cases beyond, the low-hysteresis area was determined for each configuration by testing firstly. Then, the actuation was twisted to that limit, and its behavior was recorded. The response of the actuation beyond this limit demonstrates hysteresis, and the higher the number of revolutions becomes, the bigger the hysteresis will be.

The monofilament fishing line was the first test, which was tested with two different lengths: $500 \mathrm{~mm}$ and $1,000 \mathrm{~mm}$. To acquire the result without losing generality, the results were obtained by performing each test for 50 cycles and selecting one cycle as sampled data after each consecutive 10 cycles. In this test, the experiment was including a $500 \mathrm{~g}$ loading. The utility cable and tactical cables were tested for 8 different configurations and under 4 different loadings: $500 \mathrm{~g}, 1,500 \mathrm{~g}$, 2,500 g, and $3500 \mathrm{~g}$. These tests were done 5 times for each configuration under each load in order to ensure the consistency of the response and to demonstrate the hysteresis in the response if it exists. The actuation length of the tests is set to be $500 \mathrm{~mm}$ and $1,000 \mathrm{~mm}$ anymore.

4.1. Monofilament Fishing Line. For the monofilament fishing lines (TEBCO Outcast), the rated maximum working load is $88 \mathrm{~N}$ and the diameter of each strand of the fishing line is $0.46 \mathrm{~mm}$. The shape of the filament was confirmed to be perfectly round by measuring the diameter along the length before testing. However, after several testing, not only the diameter of the string but also the overall shape of the string may be affected based on how much it was twisted or whether it was deformed beyond its flexibility range. The configuration of the string for this experiment is a twostrand string. Before it is failed due to the repetitive movements, the variation of the diameters is $0.5 \pm 0.05 \mathrm{~mm}$.

Figure 5 shows the relationship between the contracted length and motor revolution based on experimental results and theoretical calculations. It is clear that the basic model cannot precisely predict the length due to the elastic behavior caused by the additional force created by the attached weight or other factors. From the experiment, the adopted line starts to suffer from permanent distortion after 410 revolutions. The inaccuracy can be patched by the modified model with a calibrated stiffness coefficient.

To ensure the consistency of the twisted-string mechanism, the strands must be tested under repetitive motion/ force. Figure 6 demonstrates the lengths of the string of repetitive motions of both contraction and expansion for different string lengths $(500 \mathrm{~mm}$ and $1,000 \mathrm{~mm})$. From the test, the variations of contraction do not match with the trajectories of expansion, which implies that the contraction/ expansion process is nonlinear and some hysteresis exists in this type of actuation method. 


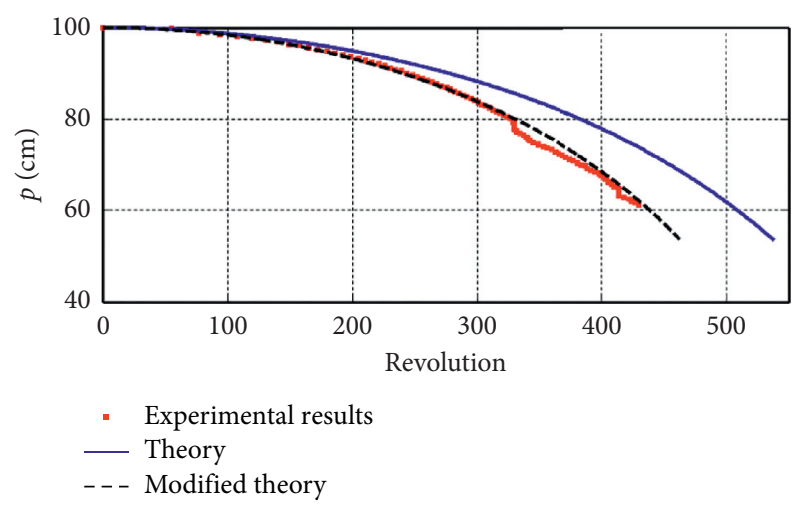

Figure 5: Comparison of the theoretical transmission length and experimental measurement for a $1,000 \mathrm{~mm}$ fishing strand.

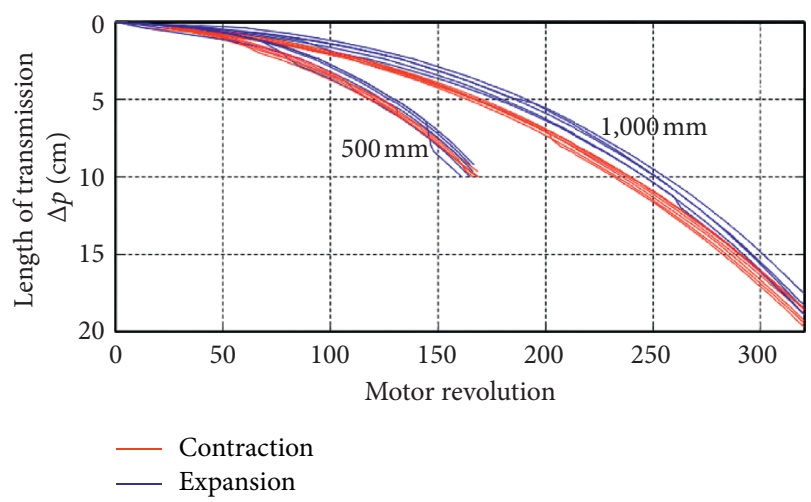

FIgURE 6: Repetitive tests of the twisted-string actuation system with two different transmission lengths $(500 \mathrm{~mm}$ and $1,000 \mathrm{~mm})$.

To investigate the hysteresis phenomenon, Figures 7 (a) and 7 (b) illustrate the contraction/expansion trajectories. Figures 7 (c) and 7(d) show the length deviations of the two processes. As shown in Figure 7, hysteresis increase as the actuator revolution increases, and after reaching a peak at half of the motor revolution, it decreases. The same trend was observed for both contraction and expansion. The maximum hysteresis for $1,000 \mathrm{~mm}$ long string is $1 \mathrm{~cm}$ and for $500 \mathrm{~mm}$ long string $0.4 \mathrm{~cm}$. If the strands were twisted more than the maximum allowed revolutions, the line begins kinking, which can yield unexpected nonlinear behavior. Kinking also distorts strands permanently and is irreversible, as shown in Figure 8. In addition, though modified model can be used to predict the contraction/expansion length, the coefficient is not a constant and can be changed when the loading is different. The friction between the two strands was not taken into consideration, either. As the revolution of the shaft increases, the friction increases as well. This part has not been included in the modified model.

4.2. Utility Line. The major drawbacks of the fishing line are its high elasticity and slow contraction. Thus, a more rigid string with higher capacity of applicable loading is desired. In this section, utility lines with $0.9 \mathrm{~mm}$ diameter and $133 \mathrm{~N}$ maximum working load was selected to work as the strands of the string.
The responses of the 2-strand actuation with and without the neutral strand are shown in Figure 9(a). Four different loading were applied to the string. They are $500 \mathrm{~g}, 1,500 \mathrm{~g}$, $2,500 \mathrm{~g}$, and $3,500 \mathrm{~g}$ masses. For each load, four repetitive experiments were performed and recorded. The twistedstring actuation has different possible motion trajectory, depending on the contraction or expansion of the strands. Plots of the motion trajectory of the twisted-string actuation often form a loop or hysteresis curve, where there are different trajectories depending on the contraction or expansion of the strands, as shown in Figures 7(a) and 7(b). In this study, the area in which the average difference between the contraction and the expansion trajectories was less than $0.5 \mathrm{~mm}$ was defined as a low-hysteresis area. The maximal allowable motor revolutions to keep the string within the low-hysteresis area for the actuation with or without neutral strand were 47 or 60 revolutions, subsequently. From the experimental result, adding a neutral strand can reduce the maximum average number of revolutions without going to the high-hysteresis area from almost 60 revolutions to 47 revolutions, which demonstrates almost $20 \%$ improvement in the actuation response. This reduction is because of the $100 \%$ increase in the effective radius $(r)$ shown in Figure 3. Moreover, the maximum contraction, within the low-hysteresis area, of the average of four tests decreased from $150 \mathrm{~mm}$ for the actuation without a neutral strand to $135 \mathrm{~mm}$ with a neutral strand, which demonstrates a $10 \%$ reduction in full contraction. From Figure 9(a), it is clear that changed length was affected greatly due to the flexibility of the string, which implies that the modified model can be a better solution as long as the stiffness coefficient can be derived. With a neutral strand existing in the string can improve the precision of actuation. The actuation without a neutral strand shows a $30 \mathrm{~mm}$ variation under different loadings for the maximum contraction, while the same configuration with a neutral strand is a $22 \mathrm{~mm}$ variation, which is a $25 \%$ reduction.

For 3-strand string actuation with and without a neutral strand, the experimental results are shown in Figure 9(b). The maximum motor revolutions within low-hysteresis area with or without neutral strand are 38 or 55 revolutions, subsequently. Similar to the previous actuation configuration, adding a neutral strand can reduce the maximum average number of revolutions (without going to the highhysteresis area) from almost 55 revolutions to 38 revolutions. This reduction is created by the $73 \%$ increase of effective radius $(r)$. The maximum contraction of the average of the four loadings decreases from $\sim 150 \mathrm{~mm}$ without a neutral strand to $\sim 110 \mathrm{~mm}$ with a neutral strand. The actuation without a neutral strand shows a $15 \mathrm{~mm}$ variation under different loadings for the maximum contraction, while the same configuration with a neutral strand is a $13 \mathrm{~mm}$ variation, which is a $13 \%$ reduction.

The responses of the actuation for 4-, 5-, and 6-strand with a neutral strand are shown in Figure 9(c). The maximum number of motor revolutions to keep actuation within low-hysteresis area is 35,31 , and 31.5 revolutions, respectively. As expected, adding effective strands can reduce maximum revolutions without yielding high-hysteresis. The 


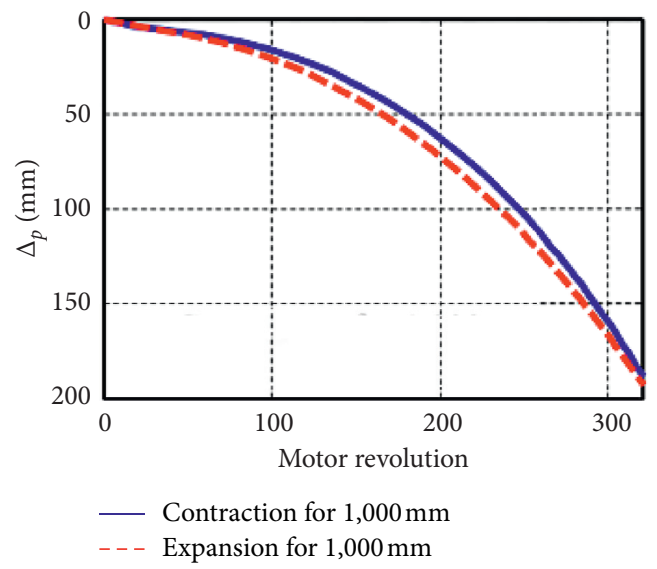

(a)

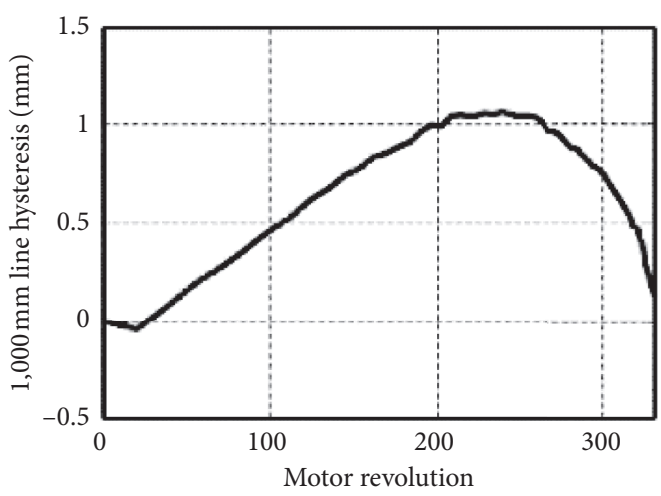

(c)

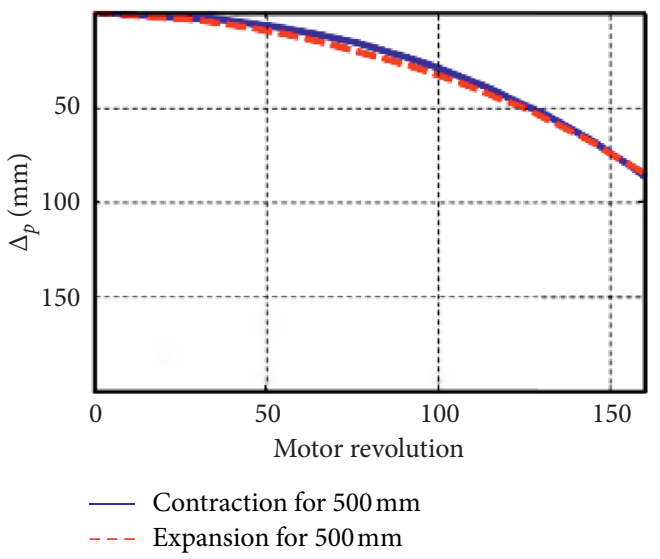

(b)

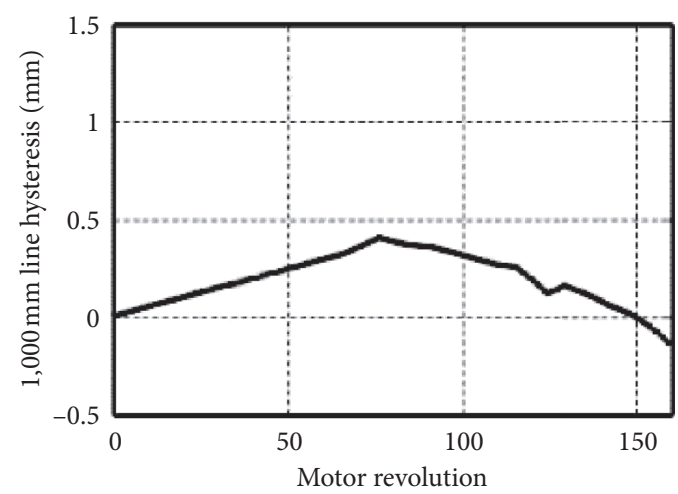

(d)

Figure 7: The changed length (fishing line) due to contraction and expansion for the original lengths of (a) 1,000 mm and (b) $500 \mathrm{~mm}$; and the deviation caused by the hysteresis of contraction relative to expansion for the lengths of (c) $1,000 \mathrm{~mm}$ and (d) $500 \mathrm{~mm}$.

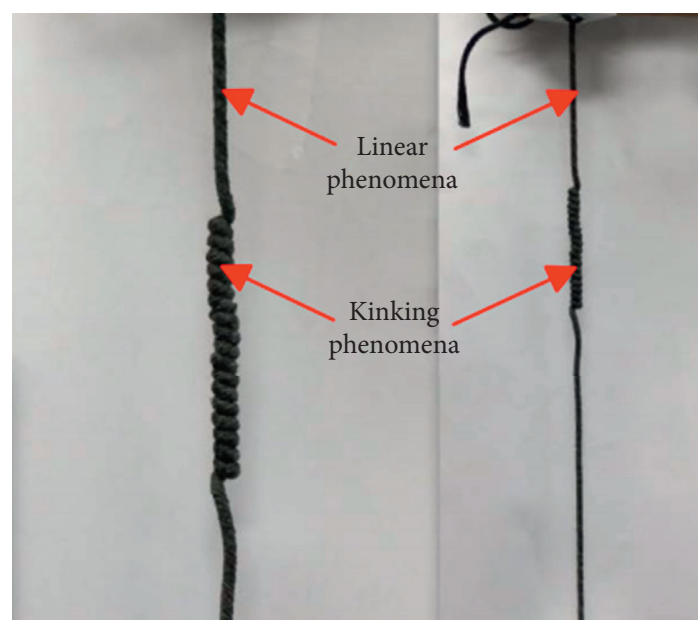

FIGURE 8: Kinking phenomena of the twisted string.

maximum contraction of the average of four tests (shown with an orange dot) is 125,125 , and $140 \mathrm{~mm}$ for the $4-, 5-$, and 6-strand configurations, respectively. All actuations demonstrate almost $\sim 13 \mathrm{~mm}$ deviation caused by the flexibility of adopted string under different loadings for the maximum contraction.
Figure 9(d) shows the comparison between a 6-strand string with and without a neutral strand. The maximum number of motor revolutions to keep the string within lowhysteresis area with and without a neutral strand is 31.5 and 31 revolutions, respectively. The maximum contraction for these two strings is $140 \mathrm{~mm}$ and $115 \mathrm{~mm}$ for the actuation with and without the neutral strand. Unlike other configurations, adding a neutral strand does not significantly change the behavior of actuation. This is mainly due to the multiple effective strand in the configuration, which greatly reduce the effectiveness of the neutral strand. However, the deviation caused by the flexibility of adopted string is not improved with the added neutral strand. From the experimental results, the deviations are both $13 \mathrm{~mm}$ under different loadings at maximum contractions. The responses of actuations for all configurations under a 3,500 g loading are shown in Figure 10. It demonstrates the change of behavior with additional strands. Table 1 summarizes the characteristics of strings with different combination of effectiveness and neutral strands. From the results, it is clear that more effective strands can make the motion less sensitive to the applied loading while reducing the maximum contraction, which can also respond faster to the changing of motor speed. 


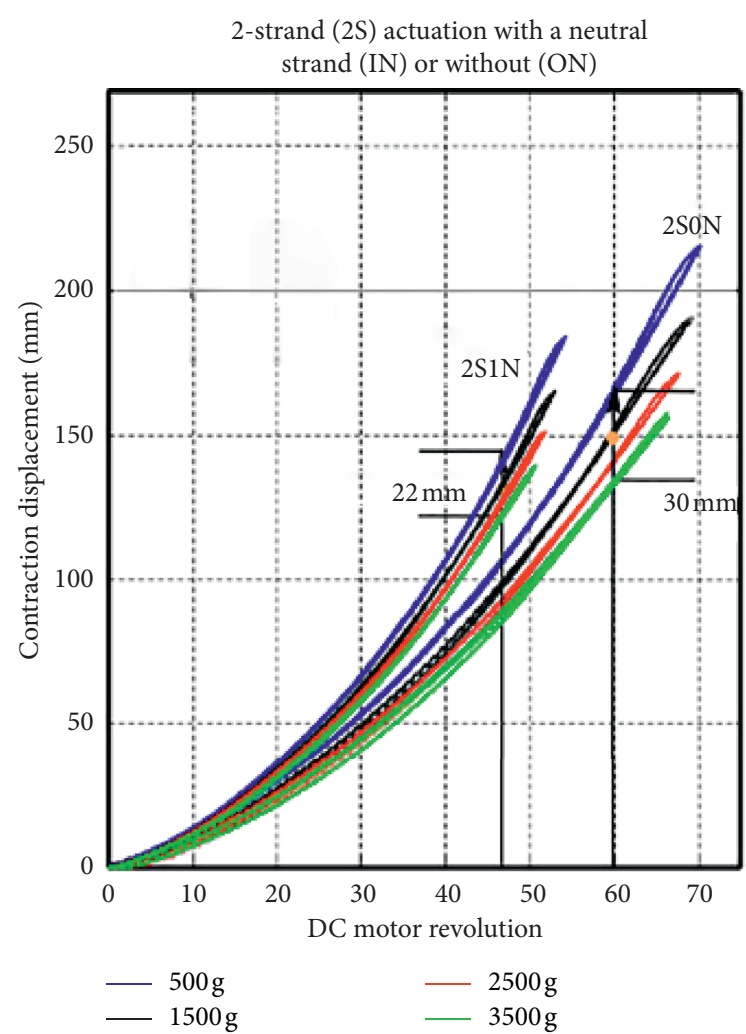

(a)

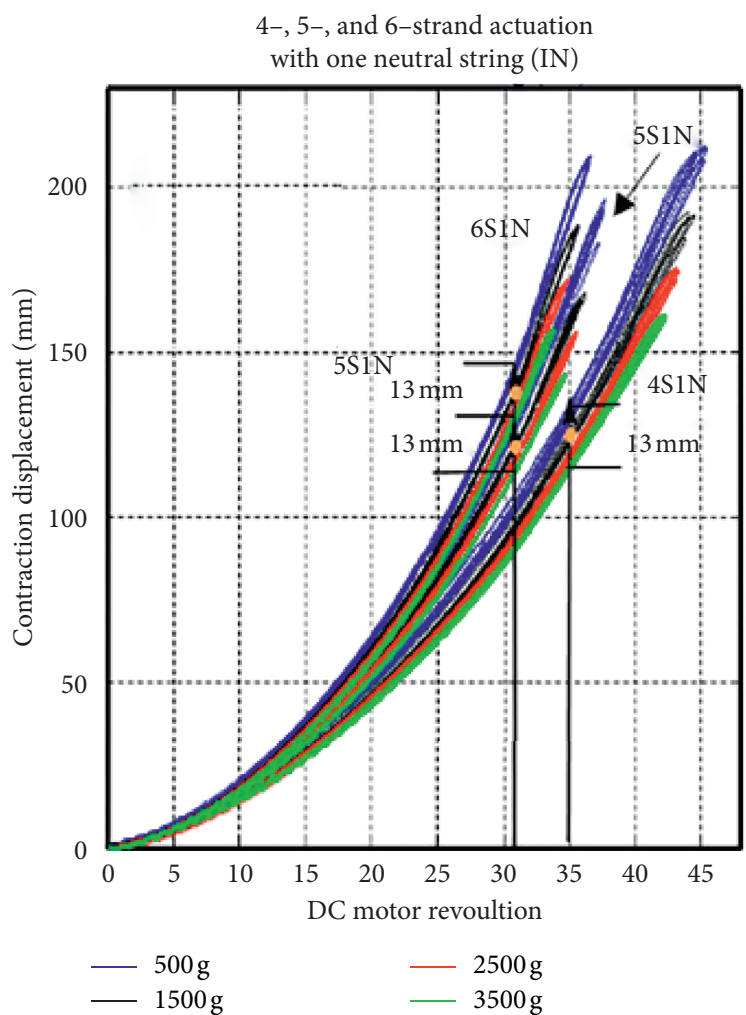

(c)

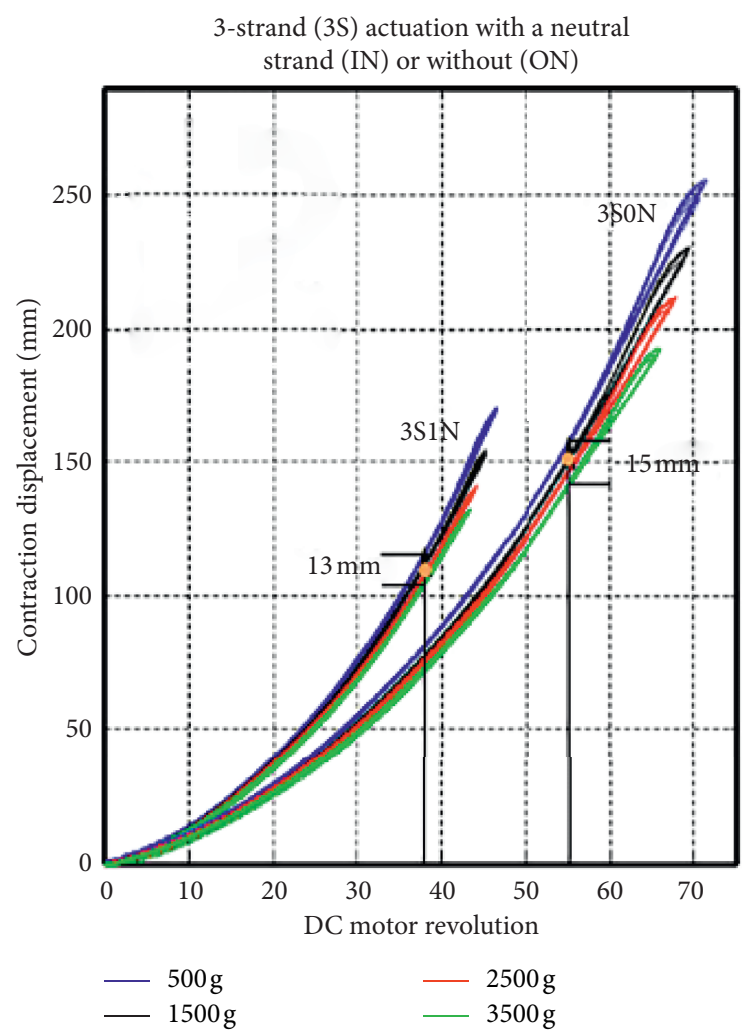

(b)

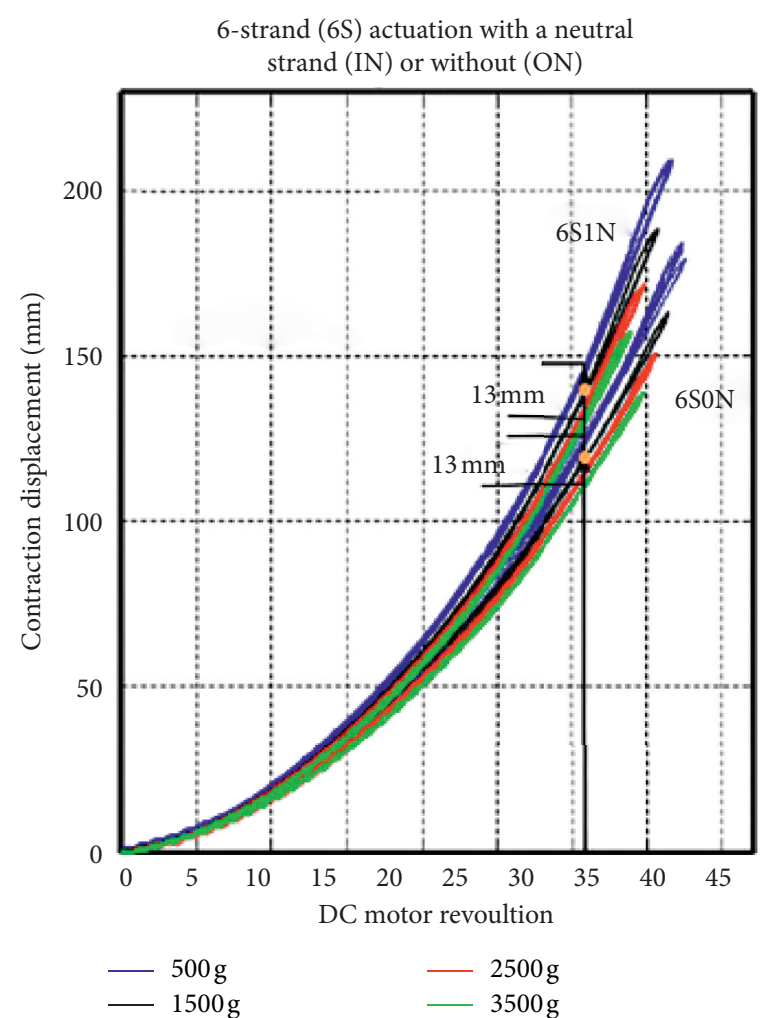

(d)

FIGURE 9: Length change of different combinations of effective strand (utility line) with or without the neutral strand: (a) 2-strand; (b) 3-strand; (c) 4-, 5-, and 6-strand; and (d) 6-strand. 


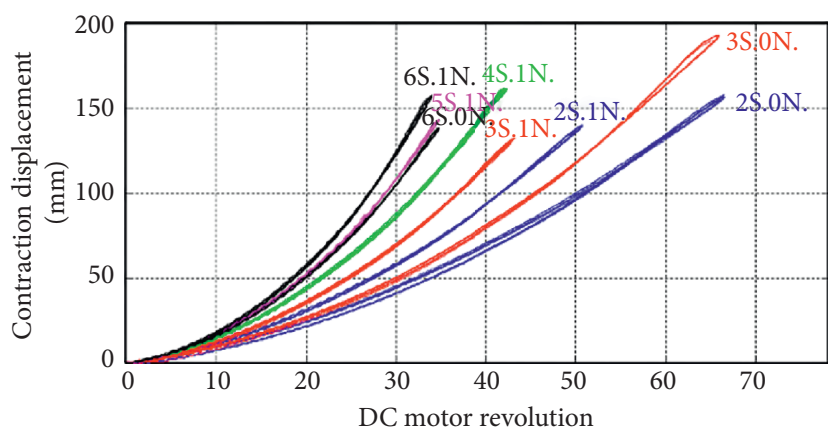

FIgURE 10: The responses of the actuation system under $3500 \mathrm{~g}$ load with different string configurations.

Table 1: Characteristics of different Configurations of utility line strings.

\begin{tabular}{|c|c|c|c|c|c|c|c|c|}
\hline Actuation type & $2 \mathrm{~S} 0 \mathrm{~N}$ & $2 \mathrm{~S} 1 \mathrm{~N}$ & $3 \mathrm{~S} 0 \mathrm{~N}$ & $3 \mathrm{~S} 1 \mathrm{~N}$ & $4 \mathrm{~S} 1 \mathrm{~N}$ & $5 \mathrm{~S} 1 \mathrm{~N}$ & $6 \mathrm{~S} 0 \mathrm{~N}$ & $6 \mathrm{~S} 1 \mathrm{~N}$ \\
\hline Max. DC motor rev & 60 & 47 & 55 & 38 & 35 & 31 & 31 & 31.5 \\
\hline Max. contraction (mm) & 150 & 135 & 150 & 110 & 125 & 125 & 115 & 140 \\
\hline Max. deviation due to loadings (mm) & 30 & 22 & 15 & 13 & 13 & 13 & 13 & 13 \\
\hline
\end{tabular}

$\mathrm{S}$ is the number of effective strands. $\mathrm{N}$ is the number of neutral strand.

4.3. Tactical Cable. Although utility line can have fast contracting response, it still does not provide accurate precision, rigidity, and resistance against load. Thus, a more rigid material with higher loading limit is desired. In this section, a tactical cable with $2.032 \mathrm{~mm}$ thickness and $200 \mathrm{~N}$ (as stated in catalog) maximum working load was adopted as the strands. This strand is made of an abrasion-resistant material (Technora), which has low stretch and high strength. Moreover, the PTFE (Teflon) coating increases the abrasion resistance and adds UV protection.

The responses of 2-strand actuation with and without a neutral strand are shown in Figure 11(a). The maximum number of motor revolutions within the low-hysteresis area for the actuation with or without neutral strand is 50 or 63 revolutions, subsequently. Adding a neutral strand can yield a $20 \%$ improvement in the actuation response for full contraction. The maximum contraction of the average of four loading tests is decreased from $165 \mathrm{~mm}$ without a neutral strand to $155 \mathrm{~mm}$ with a neutral strand $(6 \%$ reduction). The actuation without a neutral strand demonstrated $26 \mathrm{~mm}$ of variation under different loadings for maximum contraction. It is $16 \mathrm{~mm}$ with a neutral strand, which is a $38 \%$ reduction.

The responses of the 3 -strand actuation with and without the neutral strand are shown as Figure 11(b). The maximum motor revolutions within the low-hysteresis area with or without the neutral strand were 42 or 51 revolutions, respectively. Adding a neutral strand can improve the actuation response of full contraction by $19 \%$. The maximum contraction of the average of four tests is decreased from $165 \mathrm{~mm}$ without a neutral strand to $\sim 130 \mathrm{~mm}$ with a neutral strand (16\% reduction). The actuation without a neutral strand shows a $11 \mathrm{~mm}$ deviation under different loadings for its maximum contraction, while the same configuration with a neutral strand is only $9 \mathrm{~mm}$, which is an $18 \%$ reduction.

The responses of the actuation for 4,5 , and 6 effective strands and a neutral strand are shown in Figure 11(c). The maximum motor revolutions within the low-hysteresis area for the 4-, 5-, and 6-strand configurations are 36, 33, and 28 revolutions, respectively. As expected, adding effective strands can reduce the maximum average number of revolutions without going to high-hysteresis area. The maximum contraction of the average of four tests is 125,125 , and $110 \mathrm{~mm}$ for the 4-, 5-, and 6-strand strings, respectively. The maximum variation under different loadings at maximum contraction was $15 \mathrm{~mm}$ for 4-strand string actuation, $13 \mathrm{~mm}$ for 5-strand string actuation, and $9 \mathrm{~mm}$ for 6 -strand string actuation. As to 6 effective strands with and without a neutral strand, the maximum motor revolutions within lowhysteresis area are 28 and 32 revolutions, respectively. The responses of actuations for all configurations under a 3,500 g loading are shown in Figure 12. The maximum contraction of the average of four loading tests is both $110 \mathrm{~mm}$ for both actuations with or without the neutral strand. Table 2 summarizes the characteristics of different configurations using tactile cable as the strands.

As shown in Table 2, adding a neutral strand decreases the maximum number of revolutions of the DC motor, which also increases the contraction speed. To demonstrate the trend of change in the behavior of the actuation system by adding more strings, the responses under a 3,500 $\mathrm{g}$ mass loading are shown in Figure 12.

4.4. Durability of Strands. One additional critical issue of twisted-string actuation is the durability of adopted strands and wearing resistance. Figure 13 illustrates the strands of different materials after 200 working cycles. In this study, after 200 working cycles, the fishline conditions are not shown in Figure 13 because it is hard to see the difference between twisted and untwisted fishline in the picture due to the tiny size of the fishing.

As shown in Figure 13, though strands have shown some defeats after 200 working cycles, the distortions do not 


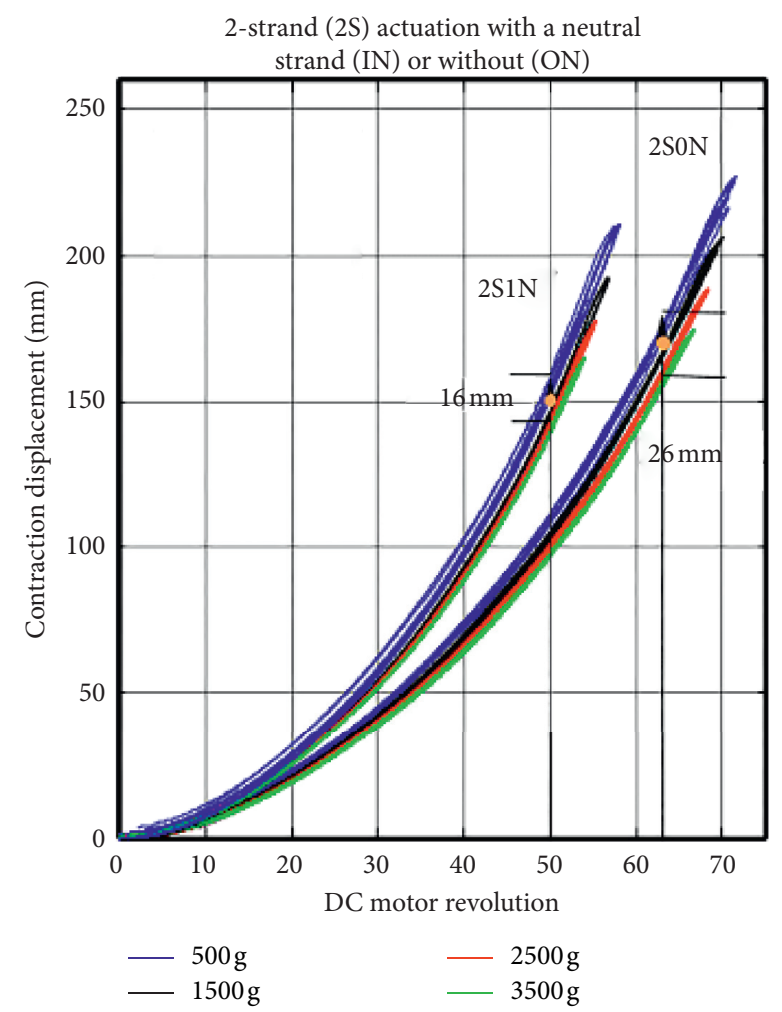

(a)

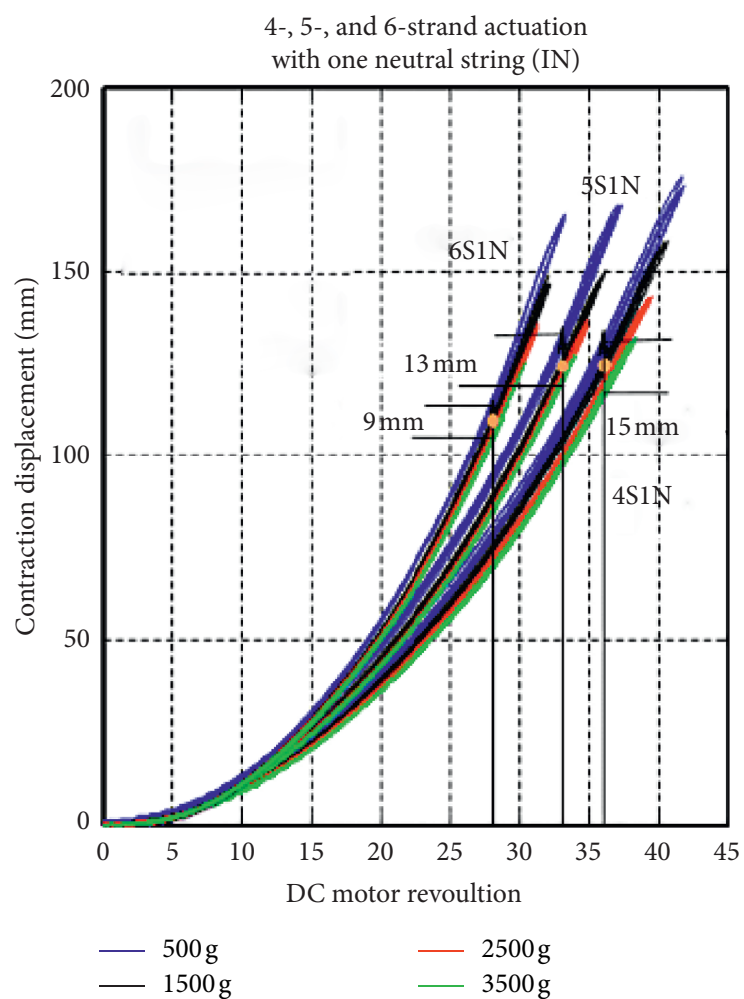

(c)

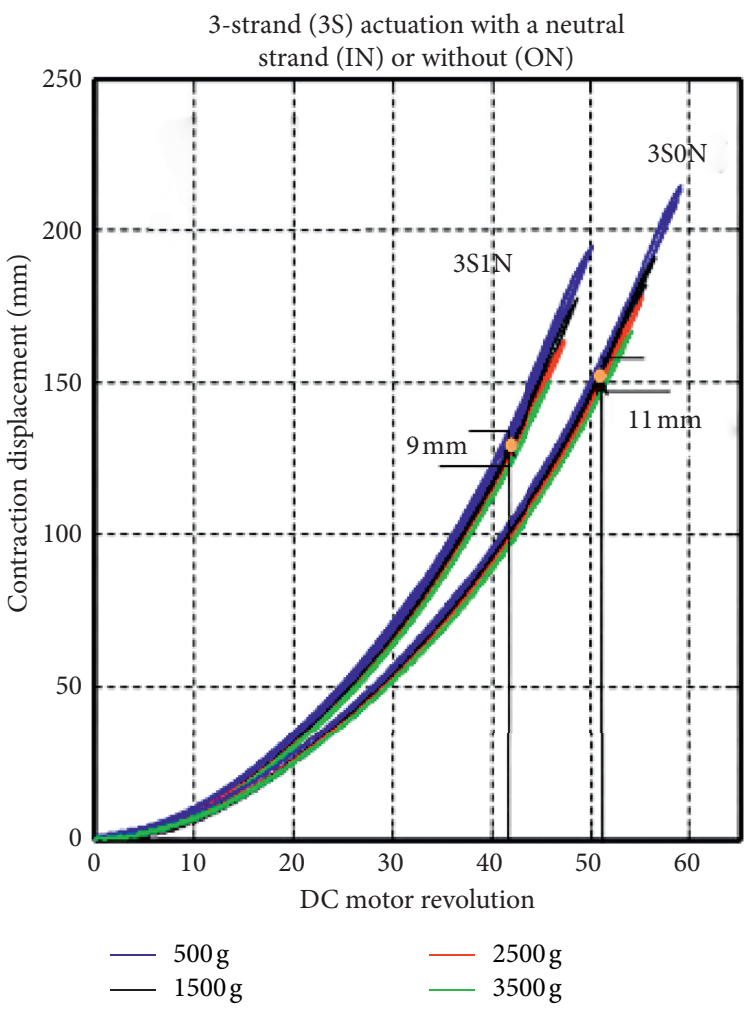

(b)

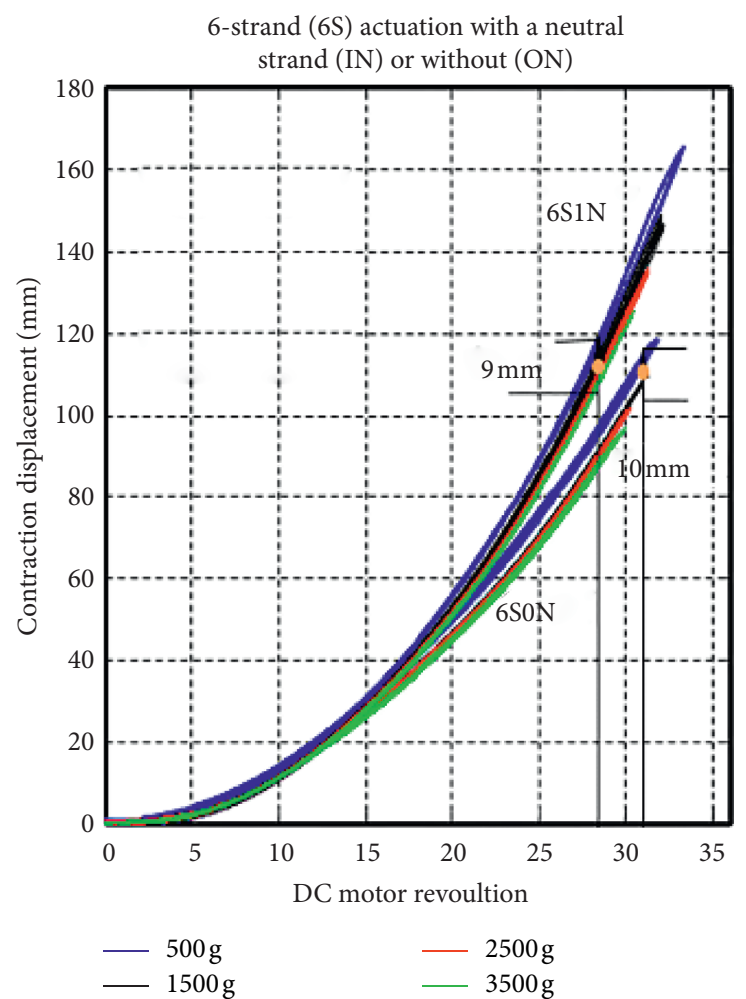

(d)

FIGURE 11: Length change of different combinations of effective strand (tactile cable) with or without the neutral strand: (a) 2-strand; (b) 3-strand; (c) 4-, 5-, and 6-strand; and (d) 6-strand. 


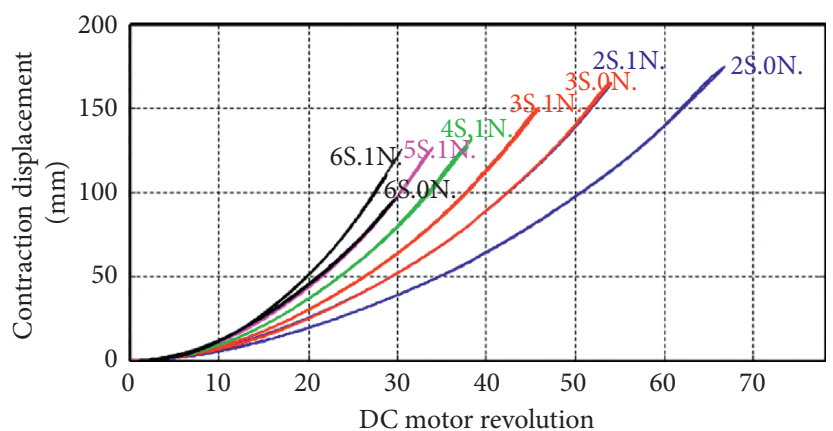

FIGURE 12: The response of the actuation system under 3,500 g mass load with different string configurations.

TABLE 2: Characteristics of different configurations of tactile cable strings.

\begin{tabular}{|c|c|c|c|c|c|c|c|c|}
\hline Actuation type & $2 \mathrm{~S} 0 \mathrm{~N}$ & $2 \mathrm{~S} 1 \mathrm{~N}$ & $3 \mathrm{~S} 0 \mathrm{~N}$ & $3 \mathrm{~S} 1 \mathrm{~N}$ & $4 \mathrm{~S} 1 \mathrm{~N}$ & $5 \mathrm{~S} 1 \mathrm{~N}$ & $6 \mathrm{~S} 0 \mathrm{~N}$ & $6 \mathrm{~S} 1 \mathrm{~N}$ \\
\hline Max. DC motor rev & 63 & 50 & 51 & 42 & 36 & 33 & 32 & 28 \\
\hline Max. contraction (mm) & 165 & 155 & 165 & 130 & 125 & 120 & 110 & 110 \\
\hline Max. deviation due to loadings $(\mathrm{mm})$ & 26 & 16 & 11 & 9 & 15 & 13 & 10 & 9 \\
\hline
\end{tabular}

$\mathrm{S}$ is the number of effective strands. $\mathrm{N}$ is the number of neutral strand.

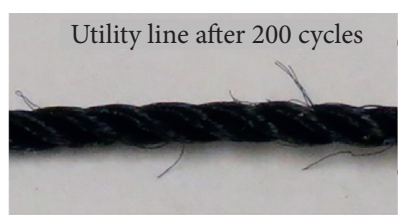

(a)

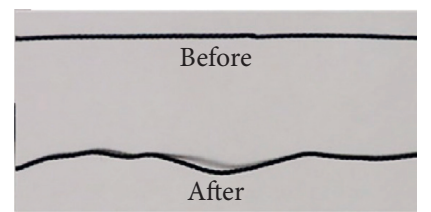

(b)

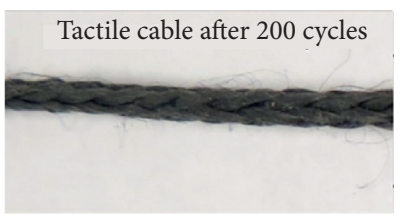

(c)

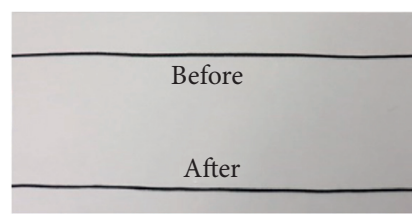

(d)

FIGURE 13: String conditions after 200 working cycles.

permanently damage the strands to affect the behavior of actuation. However, some permanent distortion can be observed. From Figure 13, it is clear that the tactile cable, which is abrasive resistive, shows much less distortion after intensive working cycles. Thus, it is suggested to check the status of the strands after certain number of working cycles. It is also important to know the lifecycle of adopted strands.

In addition to available lifecycle, some inappropriate operations can damage the strands as well. One most common operation is to rotate the twisted-string beyond the allowable range of adopted strands. While being operated beyond the allowable range, the string becomes kinking because it is over twisted. Once the string becomes kinking, the string can suffer from irreversible damage. However, the nonlinear area covers the response of the actuation after going to the unpredictable nonlinear range. The distribution of low-hysteresis, high-hysteresis, and nonlinear areas is shown in Figure 14.

\section{ARC Control Synthesis of the Twisted String Actuation}

Twisted-string actuation has the advantages of compact size, flexible mechanism, and lightweight at locations where actuations are required. However, though twisted-string actuation has many advantages, its unique properties of nonlinearity including hysteresis, friction, operational dead-

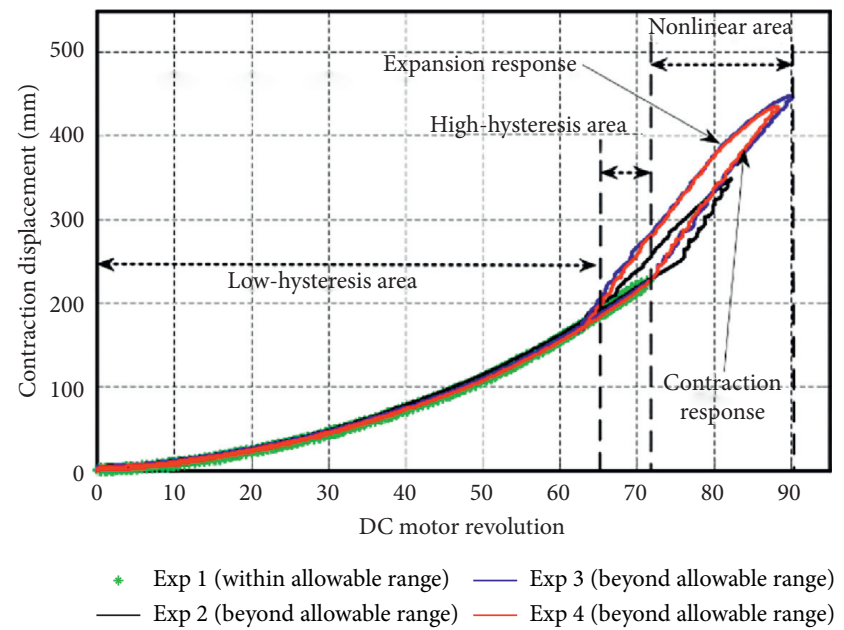

FIgURE 14: The response of a 2-stand string actuation in lowhysteresis, high-hysteresis, and nonlinear areas.

zone, and poor repeatability can deteriorate the desired control performance. Improper control methods without consideration of nonlinearities can lead to limit cycles or instability. To deal with both nonlinear factors, adaptive robust control (ARC) was adopted for such a motion system. Although this kinetostatic model described in the last section can reflect the relationship between axial forces, torque 
generated by motor, and string length, there are still obvious differences between mathematical modeling and actual performance of a twisted string. The main reason for the differences can be caused by not considering of friction among strands, the gap among different strands, elasticity of single strands, and deformation of individual strands. Therefore, the twisted-string actuation system falls into the class of the real-world processes that are not easy to obtain the mathematical representation. In real-time control system, there is a large variety of plants, similar to twisted-string actuator, whose system model is highly nonlinear and cannot be physically derived. Figure 14 demonstrates the relationship between motor revolutions and force generated by the twisted-string actuator. Since the force transmission of the twisted-string actuator plays the key role in controller design, the constant current source is used to drive the DC motor, which can ensure the torque generated by DC motor stays unchanged during the whole process of twisting string. The data, as shown in Figure 15, are obtained when the constant current source provides 0.7 Amp current. From Figure 15(a), it is obvious that the force transmission keeps increasing along with motor revolutions when the torque generated by the DC motor is constant, which means that the force transmission efficiency can be increased by pretwisting string to specify operation range. The two stages of transition are shown in Figures 15(a) and 15(b), and the two phases of the force transmission are initial phase, in which the twisted-string actuator does not generate the linear displacement, and normal phase. It is easy to make a conclusion that the force transmission will increase to infinity based on simply observing Figure 15(a); however, with the increasing of motor revolutions, the parts of torque generated by the DC motor used to overcome the friction among strands will also be increased.

Based upon above experimental results and above analysis, it is clear that if we pretwist string before using the twisted-string actuator to drive the external load, the hysteresis effect, time delay, and the irregular force transmission in the initial phase can be avoided, which is very important for designing a high-accuracy controller of motion systems. Five experiments are performed with the goal of verifying this assumption. In these five experiments, the string is pretwisted five turns before the experiments starting. Figure 16 illustrates the relationship among motor revolutions, force, and linear displacements generated by the twistedstring actuator after pretwisting string. Both the force and linear displacement curves express better continuity than no pretwisted curves, as shown in Figure 16. Therefore, pretwisting the string is a method, efficient and easy to achieve, to reduce the complexity of controller design. Although this kinetostatic model described in the last section can reflect the relationship between axial forces, the torque generated by motor, and string length, there are still obvious differences between mathematical modeling and actual performance of a twisted string. For these nonlinear plants, the experiments can be performed to extract approximate model by using sophisticated curve fitting approaches. In this study, the approximation of the static characteristic of the twist string actuator is made from Figure 17 using the Matlab toolbox called Curve Fitting Tool. The armature current of DC motor which is proportional to torque is the input of the motion system in this study. Therefore, the function of force transmission and linear displacements are required to design the controller. The approximate function of force transmission and linear displacement are found by the curve fitting approach. Then, the force generated by the twisted-string actuator can be predicted by this approximate function. The approximate function is exponentiation function:

$$
K_{\tau}(x)=\alpha+\beta \cdot e^{-\gamma x} .
$$

The free-body diagram for the load is shown in Figure 2. The equation of motion of the mass $m$ is

$$
m \ddot{x}=F-m g-F_{L}-f+\Delta,
$$

where $m$ is the mass of the load, $F$ is the tensile force generated by the twisted-string actuator, $x$ is the displacement of the load, $F_{L}$ is the external force, which can be measured by the force sensor, $f$ is the friction among strands, and $\Delta$ is unstructured uncertainties of the model. Based on equation (11), we can rewrite equation of motion (12) as follows:

$$
m \ddot{x}=\frac{\tau_{l}}{K_{\tau}\left(x_{1}\right)}-m g-F_{L}-f+\Delta,
$$

where $K_{\tau}$ is the relationship between the displacement of the load and the tensile force generated by twisted-string actuator and $\tau_{L}$ is the torque generated by the DC motor, which is equal:

$$
\tau_{l}=K_{t} I
$$

where $K_{t}$ is the torque constant of the DC motor and $I$ is the armature current of the DC motor. We can build the dynamic model of the twisted-string actuation system in a state-space form as follows:

$$
\begin{aligned}
& \dot{x}_{1}=x_{2} . \\
& \dot{x}_{2}=\frac{1}{m}\left(\frac{\tau_{l}}{K_{\tau}\left(x_{1}\right)}-m g-F_{L}-f+\Delta\right),
\end{aligned}
$$

where $x=\left[\begin{array}{ll}x_{1} & x_{2}\end{array}\right]^{\mathrm{T}}=\left[\begin{array}{ll}x & \dot{x}\end{array}\right]$ is the system state. The traditional velocity and position controller design method focuses on the mechanical dynamics with an inertial load but ignoring the electrical dynamics. With the aim of high tracking performance, it is required to consider the electrical system and the connection between the electrical system and mechanical systems. Form Figure 2, it is obvious that the control input voltage does not directly influence the displacement of the load. To cope with this, the concept of backstepping can be employed to deal with the first equation in (15) not including the control input. We can rewrite the state-space model by integrating the electrical subsystem as follows:

$$
\begin{aligned}
& \dot{x}_{1}=x_{2}, \\
& \dot{x}_{2}=\frac{1}{m}\left(\frac{K_{t} I}{K_{\tau}\left(x_{1}\right)}-m g-F_{L}-f+\Delta\right), \\
& L \dot{I}=u-\mathrm{RI}-K_{b} \omega,
\end{aligned}
$$




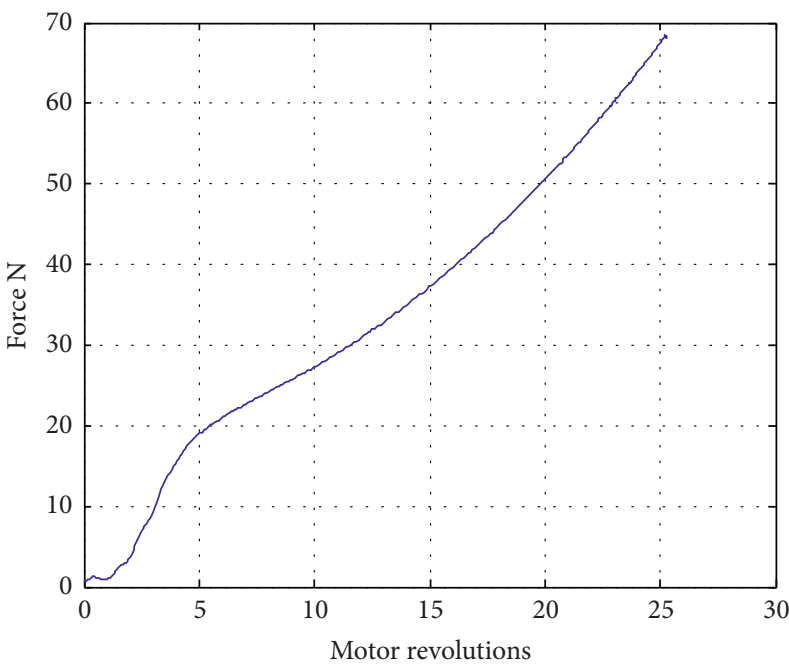

(a)

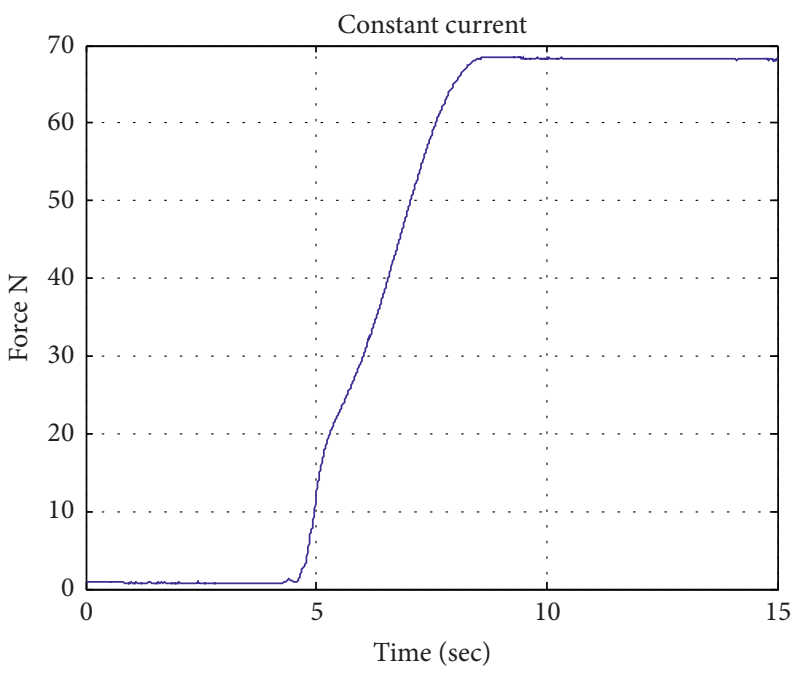

(b)

FIGURE 15: Relationship between motor revolutions and force.

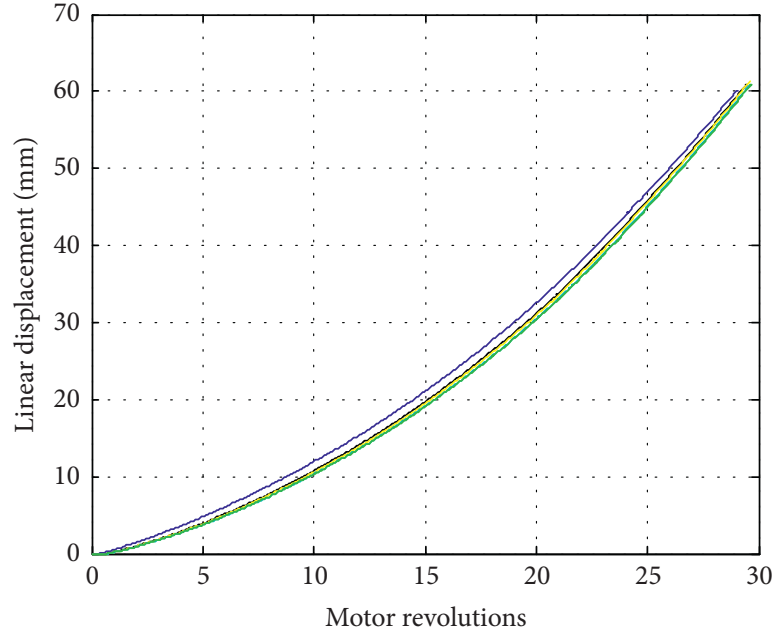

Experiment 1
- Experiment 2
Experiment 3

- Experiment 4

- Experiment 5

(a)

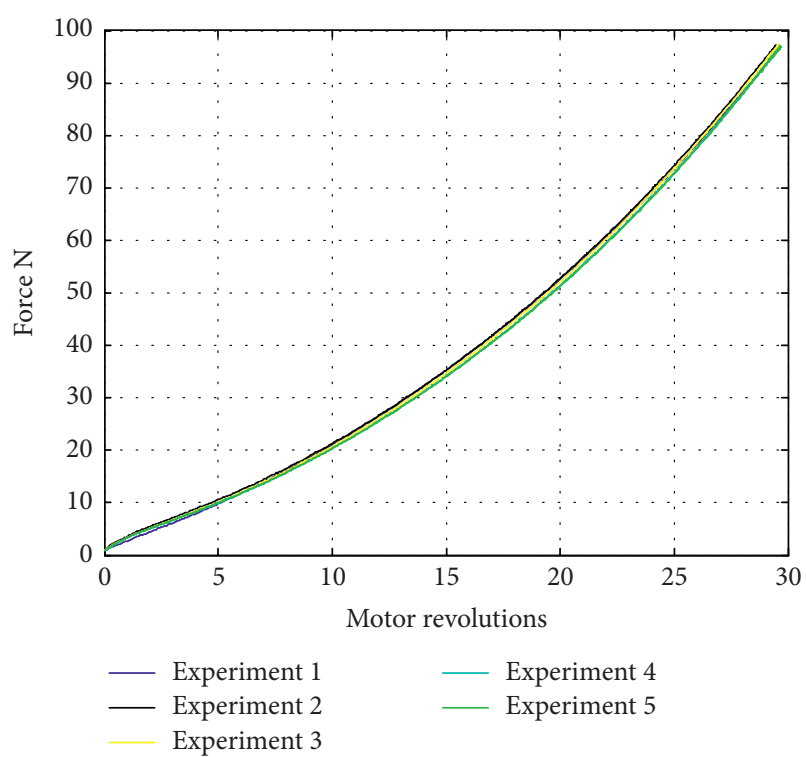

(b)

FIGURE 16: Relationship among motor revolutions, force, and stroke with pretwist.

where $L$ is the constant rotor inductance, $R$ is the constant rotor resistance, $K_{b}$ is the constant back EMF coefficient, $\omega$ is the speed of DC motor, and $u$ is the input control voltage. Define a switch function, which is original from slide mode control, as

$$
\begin{aligned}
Z & =\dot{e}+k_{1} e, \\
e & =x_{1 d}-x_{1},
\end{aligned}
$$

where $e$ is error between desired trajectory and actual trajectory of external load's displacement, and $k_{1}$ positive feedback gain. The transfer function form $Z$ to $e$ is

$$
E(s)=\frac{1}{s+k_{1}} Z(s)
$$

Since the $Z(s)$ is a linear system and $k 1$ is positive, the $e(t)$ will be converging zero if the $Z(t)$ converges to zero. Then, the $E(s)$ will be converging to zero if the $Z(t)$ and $e(t)$ are converging to zero. View the state variable $I$ as an independent virtual "input." The first step of the controller design is to ensure this virtual "input" can both stabilizes the second equation in (15) and let the $Z(t)$ eventually goes to zero, which guarantee that the tacking error $e(t)$ converges to zero. Differentiating $Z$ with respect to time leads to 


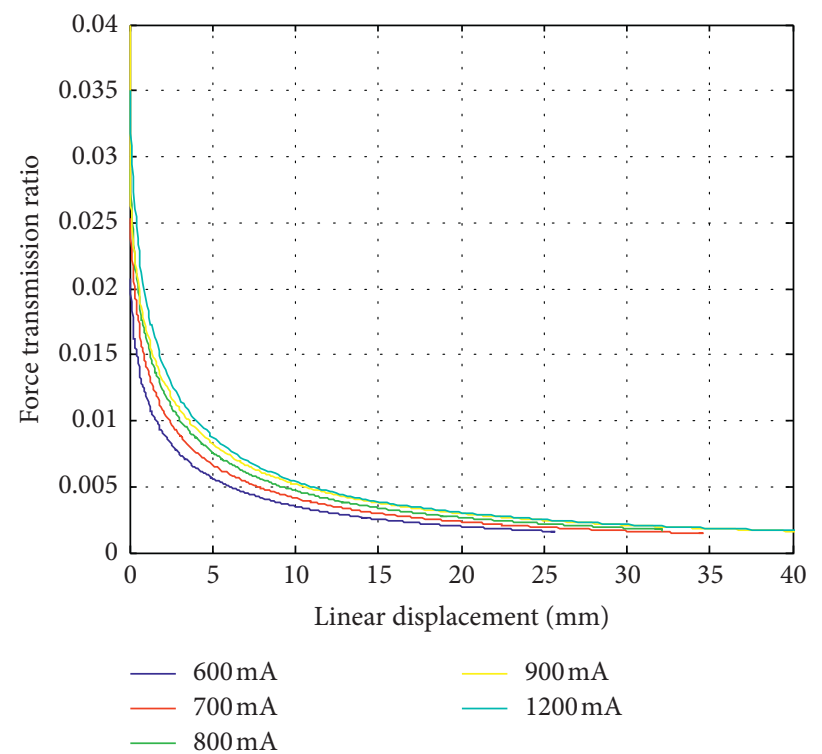

FIGURE 17: Relationship among force transmission ratio and linear displacements after pretwisting.

$$
\dot{Z}=\ddot{x}_{1 d}-\ddot{x}_{1}+k_{1} \dot{e} .
$$

By combing the second equation in (11), we have

$$
\dot{Z}=\ddot{x}_{1 d}-\frac{1}{m}\left(\frac{K_{t} I}{K_{\tau}\left(x_{1}\right)}-m g-F_{L}-f+\Delta\right)+k_{1} \dot{e} .
$$

Rewrite (19) as

$$
\begin{aligned}
\frac{K_{\tau}\left(x_{1}\right) m}{K_{t}} \dot{Z}= & \frac{K_{\tau}\left(x_{1}\right) m_{\ddot{x}_{1 d}}-\left(I-\frac{K_{\tau}\left(x_{1}\right)\left(m g+F_{L}+f-\Delta\right)}{K_{t}}\right)}{K_{t}} \\
& +\frac{K_{\tau}\left(x_{1}\right) m}{K_{t}} k_{1} \dot{e} .
\end{aligned}
$$

Substitute (11) into (21):

$$
\frac{\left(\alpha+\beta e^{-\gamma x_{1}}\right) m}{K_{t}} \dot{Z}=\frac{\left(\alpha+\beta e^{-\gamma x_{1}}\right) m}{K_{t}} \ddot{x}_{1 d}-\left(I-\frac{\left(\alpha+\beta e^{-\gamma x_{1}}\right)\left(m g+F_{L}+f-\Delta\right)}{K_{t}}\right)+\frac{\left(\alpha+\beta e^{-\gamma x_{1}}\right) m}{K_{t}} k_{1} \dot{e} .
$$

Rewrite (22) as

$$
p\left(x_{1}\right) \dot{Z}=\mathbf{W} \boldsymbol{\theta}-I,
$$

where $\quad \mathbf{W}=\left[\left(m / K_{t}\right)\left(\ddot{x}_{1 d}+k_{1} \dot{e}+g\right)\left(m / K_{t}\right) e^{-\gamma x_{1}}\left(\ddot{x}_{1 d}+k_{1}\right.\right.$ $\left.\dot{e}+g)\left(1 / K_{t}\right)\left(e^{-\gamma x_{1}} / K_{t}\right)\right], \quad \theta=\left[\alpha \beta \alpha\left(F_{L}+f-\Delta\right) \beta\left(F_{L}+\right.\right.$ $f-\Delta)]^{T}, p\left(x_{1}\right)=\left(\left(\left(a+b e^{-\gamma x_{1}}\right) m\right) / K_{t}\right)$.

The adaptive robust control mechanism compensate the unknown parameters and structured uncertainties by adjusting parameters online. However, even a small disturbance may lead the parameters adaptive process to instability and large transient tracking error. By integrating the essence of the deterministic robust control (DRC), the adaptive robust controller can attenuate the effect of unstructured uncertainties and improve the transient performance. Therefore, the desired virtual input $I$ can be given by

$$
\begin{gathered}
I=I_{a}+I_{s}, \\
I_{s}=I_{s 1}+I_{S 2},
\end{gathered}
$$

where $I_{a}$ is adjustable feedforward controller used to compensate the model by parameter adaption, $I_{s}$ is the robust controller in which $I_{s 1}$ is linear feedback to stabilize the nominal model of the twisted-string actuation and $I_{s 2}$ is used to attenuate the parameter estimation error and nonlinear term of model. In equation (23), the vector $\boldsymbol{\theta}$ are all unknown parameters that need to be estimated. Therefore, the vector $\boldsymbol{\theta}$ will be replaced by the $\widehat{\theta}$, the estimated value of the vector $\boldsymbol{\theta}$, in the controller. Since the $K_{\tau}\left(x_{1}\right)$ and $K_{t}$ are positive function and constant, the Lyapunov candidate function can be defined as follows:

$$
V=\frac{1}{2} p\left(x_{1}\right) Z^{2}+\frac{1}{2} \widetilde{\theta}^{\mathrm{T}} \widetilde{\theta},
$$

where $\widetilde{\theta}$ is the difference between the real value of the vector $\boldsymbol{\theta}$ and $\hat{\theta}$, the estimated value of the vector. Taking the derivative of (25) gives

$$
\dot{V}=\frac{1}{2} \dot{p}\left(x_{1}\right) Z^{2}+p\left(x_{1}\right) Z \dot{Z}+\widetilde{\theta}^{\mathrm{T}} \dot{\tilde{\theta}} .
$$

Since the $\dot{\tilde{\theta}}=\dot{\theta}-\dot{\hat{\theta}}$ and $\theta$ is constant, we have

$$
\dot{V}=\frac{1}{2} \dot{p}\left(x_{1}\right) Z^{2}+p\left(x_{1}\right) Z \dot{Z}-\tilde{\theta}^{\mathrm{T}} \dot{\hat{\theta}} .
$$

Substituting (23) into (27) gives 


$$
\dot{V}=\frac{1}{2} \dot{p}\left(x_{1}\right) Z^{2}+Z(\mathbf{W} \boldsymbol{\theta}-I)-\widetilde{\theta}^{\mathrm{T}} \dot{\hat{\theta}}
$$

Since the $I_{a}$ is adjustable feedforward controller used to compensate the model by parameter adaption, the $I_{a}$ is

$$
I_{a}=\mathbf{W} \widehat{\theta}+\frac{1}{2} \dot{p}\left(x_{1}\right) Z
$$

Letting $I_{s 1}=K Z$, we get

$$
\dot{V}=-K Z^{2}+Z \mathbf{W} \widetilde{\theta}-\widetilde{\theta}^{\mathrm{T}} \dot{\hat{\theta}}
$$

where $K$ is a positive feedback gain. In order to ensure the stability of the system, the derivative of $V$ should be less or equal to zero by letting

$$
\mathbf{Z W} \tilde{\theta}-\tilde{\theta}^{\mathrm{T}} \dot{\hat{\theta}}=0
$$

Solving equation (31) gives

$$
\dot{\hat{\theta}}=\mathbf{W}^{\mathrm{T}} Z \text {, }
$$

where $\quad \dot{\hat{\theta}}=\left[\begin{array}{llll}\dot{\hat{\theta}}_{1} & \dot{\hat{\theta}}_{2} & \dot{\hat{\theta}}_{3} & \dot{\hat{\theta}}_{4}\end{array}\right]$. Therefore, the adaptation mechanisms for adjusting parameters are

$$
\begin{aligned}
& \dot{\hat{\theta}}_{1}=\frac{m}{K_{t}}\left(\ddot{x}_{1 d}+k_{1} \dot{e}+g\right) Z, \\
& \dot{\hat{\theta}}_{2}=\frac{m}{K_{t}} e^{-c x_{1}}\left(\ddot{x}_{1 d}+k_{1} \dot{e}+g\right) Z, \\
& \dot{\hat{\theta}}_{3}=\frac{1}{K_{t}} Z, \\
& \dot{\hat{\theta}}_{4}=\frac{e^{-c x_{1}}}{K_{t}} Z .
\end{aligned}
$$

Assuming the error between the actual virtual input and the desired virtual input is

$$
e_{2}=I-I_{d}
$$

where the $I$ is the actual virtual input and $I_{d}$ is the desired virtual input.

Substituting (34) into (23) gives

$$
p\left(x_{1}\right) \dot{Z}=\mathbf{W} \boldsymbol{\theta}-\left(e_{2}+I_{d}\right) \text {. }
$$

Substituting (29) into (35) gives

$$
p\left(x_{1}\right) \dot{Z}=\mathbf{W} \boldsymbol{\theta}-\left(e_{2}+\mathbf{W} \hat{\theta}+\frac{1}{2} \dot{p}\left(x_{1}\right) Z+K Z+I_{S 2}\right) \text {. }
$$

Rewrite (36) as

$$
p\left(x_{1}\right) \dot{Z}=-e_{2}-\frac{1}{2} \dot{p}\left(x_{1}\right) Z-K Z+\mathbf{W} \tilde{\theta}-I_{S 2} .
$$

For the deterministic robust control, the robust term $I_{s 2}$ should be any continuous function which satisfies the following conditions:

$$
\begin{aligned}
& \text { (1) } Z\left(\mathbf{W} \widetilde{\theta}-I_{S 2}\right) \leq \varepsilon, \\
& \text { (2) } Z I_{S 2} \geq 0
\end{aligned}
$$

where $\varepsilon$ is a positive design parameter that can be set arbitrarily small. Furthermore, the Lyapunov function can be defined as follows:

$$
V_{s}=\frac{1}{2} p\left(x_{1}\right) Z^{2}
$$

The derivative of the $V_{s}$ becomes

$$
\dot{V}_{s}=\frac{1}{2} \dot{p}\left(x_{1}\right) Z^{2}+p\left(x_{1}\right) Z \dot{Z} .
$$

One example of $I_{s 2}$ satisfying equation (38) is

$$
I_{s 2}(t)=Z \frac{\left(\mathbf{W} \boldsymbol{\theta}_{m}\right)^{2}}{\varepsilon},
$$

where $\theta_{m}=\theta_{\max }-\theta_{\min }, \theta_{\max }$ is the upper limit of the $\theta$, and $\theta_{\min }$ is the lower limit of the $\theta$. Substituting (6) and (10) into (9) gives

$$
\dot{V}_{s}=-Z e_{2}-K Z^{2}+Z\left(\mathbf{W} \widetilde{\theta}-Z \frac{\left(\mathbf{W} \boldsymbol{\theta}_{m}\right)^{2}}{\varepsilon}\right) .
$$

From the definition of the $\theta_{m}$, the derivative of the $V_{s}$ satisfies

$$
\dot{V}_{s} \leq-Z e_{2}-K Z^{2}+|Z|\left|\mathbf{W} \boldsymbol{\theta}_{m}\right|\left(1-|Z| \frac{\left|\mathbf{W} \boldsymbol{\theta}_{m}\right|}{\varepsilon}\right) .
$$

Case 1: if $|Z|\left|\mathbf{W} \theta_{m}\right|>\varepsilon$, then

$$
\begin{aligned}
|Z| \frac{\left|\mathbf{W} \boldsymbol{\theta}_{m}\right|}{\varepsilon}>1 & \longrightarrow\left(1-|Z| \frac{\left|\mathbf{W} \boldsymbol{\theta}_{m}\right|}{\varepsilon}\right)<0 \\
& \longrightarrow|Z|\left|\mathbf{W} \boldsymbol{\theta}_{m}\right|\left(1-|Z| \frac{\left|\mathbf{W} \boldsymbol{\theta}_{m}\right|}{\varepsilon}\right)<0 \\
& \Rightarrow \dot{V}_{s} \leq-Z e_{2}-K Z^{2} .
\end{aligned}
$$

Case 2: if $|Z|\left|\mathbf{W} \theta_{m}\right|<\varepsilon$, then

$$
\begin{aligned}
|Z| \frac{\left|\mathbf{W} \boldsymbol{\theta}_{m}\right|}{\varepsilon}<1 & \longrightarrow 0 \leq\left(1-|Z| \frac{\left|\mathbf{W} \boldsymbol{\theta}_{m}\right|}{\varepsilon}\right)<1 \\
& \longrightarrow|Z|\left|\mathbf{W} \boldsymbol{\theta}_{m}\right|\left(1-|Z| \frac{\left|\mathbf{W} \boldsymbol{\theta}_{m}\right|}{\varepsilon}\right) \leq|Z|\left|\mathbf{W} \boldsymbol{\theta}_{m}\right| \\
& \Rightarrow \dot{V}_{s} \leq-Z e_{2}-K Z^{2}+\varepsilon .
\end{aligned}
$$

In the first case, assuming $e_{2}=0$, the inequality (44) then becomes

$$
\dot{V}_{s} \leq-K Z^{2} \leq-2 \frac{K}{p\left(x_{1}\right)} V_{s}
$$


The following inequality then can be derived by integrating (46):

$$
V_{s}(t) \leq \exp \left(\frac{-2 K t}{\left\|p\left(x_{1}\right)\right\|_{\max }}\right) V_{s}(0),
$$

where $t$ is time variant, and $\left\|p\left(x_{1}\right)\right\|_{\max }$ is the upper bound of $p\left(x_{1}\right)$. According to Bellman-Gronwall, the positive definite $V_{s}$ is bounded by

$$
V_{s}(t) \leq \exp \left(\frac{-2 K t}{\left\|p\left(x_{1}\right)\right\|_{\max }}\right) V_{s}(0) .
$$
by

Based on the relationship between $Z$ and $e, e$ is bounded

$$
e(t) \leq \exp (-k t) \cdot e(0)
$$

In the second case, assuming the $e_{2}=0$, inequality (45) then becomes

$$
\dot{V}_{s} \leq-K Z^{2}+\varepsilon \leq-2 \frac{K}{p\left(x_{1}\right)} V_{s}+\varepsilon .
$$

The following inequality then can be derived by integrating (50). That is,

$$
V_{s}(t) \leq \exp \left(\frac{-2 K t}{\left\|p\left(x_{1}\right)\right\|_{\max }}\right) V_{s}(0)+\int_{0}^{t} \exp \left(\frac{-2 K(t-v)}{\left\|p\left(x_{1}\right)\right\|_{\max }}\right) \varepsilon \mathrm{d} v,
$$

where $t$ is time variant, and $\left\|p\left(x_{1}\right)\right\|_{\max }$ is the upper bound of $p\left(x_{1}\right)$. According to Bellman-Gronwall, the positive definite $V_{s}$ is bounded by

$$
\begin{aligned}
V_{s}(t) \leq & \exp \left(\frac{-2 K t}{\left\|p\left(x_{1}\right)\right\|_{\max }}\right) V_{s}(0) \\
& +\frac{\varepsilon\left\|p\left(x_{1}\right)\right\|_{\max }}{2 K}\left[1-\exp \left(\frac{-2 K t}{\left\|p\left(x_{1}\right)\right\|_{\max }}\right)\right] .
\end{aligned}
$$
by

Based on the relationship between $Z$ and $e, e$ is bounded

$$
e(t) \leq \exp \left(-k_{1} t\right) \cdot e(0)+\frac{Z}{k_{1}}\left[1-\exp \left(-k_{1} t\right)\right]
$$

From the above inequalities, the tracking error of the twisted-string system exponentially approaches to a ball with converging rate $k_{1}$. Within a finite duration, the asymptotic output tracking is achieved. If we know the error between the actual virtual input and desired virtual input goes to zero, we can stabilized the $e$. Therefore, the next objective of the controller design is to ensure the $e_{2}$ approaches to zero. Taking the derivative of the $e_{2}$ gives

$$
\dot{e}_{2}=\dot{I}-\dot{I}_{d}
$$

Substituting the third equation in (16) into (54) gives

$$
\dot{e}_{2}=\frac{u-\mathrm{RI}-K_{b} \omega}{L}-\dot{I}_{d}
$$

Since $I_{d}$ is designed as $I_{d}=I_{a}+I_{s 1}+I_{s 2}$, then

$$
\begin{aligned}
\dot{I}_{d} & =\dot{I}_{a}+\dot{I}_{s 1}+\dot{I}_{s 2} \\
& =\frac{\mathrm{d}}{\mathrm{d} t}\left(\mathbf{W} \widehat{\theta}+\frac{1}{2} \dot{p}\left(x_{1}\right) Z\right)+K \dot{Z}+\dot{I}_{s 2} \\
& =\mathbf{W} \dot{\hat{\theta}}+\frac{1}{2} \ddot{p}\left(x_{1}\right) Z+\frac{1}{2} \dot{p}\left(x_{1}\right) \dot{Z}+K \dot{Z}+\dot{I}_{s 2} .
\end{aligned}
$$

Rewrite (55) as

$$
L \dot{e}_{2}=u-\mathbf{W}_{1} \boldsymbol{\theta}_{1},
$$

where $\mathbf{W}_{1}=\left[\begin{array}{lll}I & \omega & \dot{I}_{d}\end{array}\right], \theta_{1}^{\mathbf{T}}=\left[\begin{array}{lll}R & k_{b} & L\end{array}\right]$.

Using the same design procedure as in the above, the input $u$ also can be given by

$$
\begin{gathered}
u=u_{a}+u_{s} . \\
u_{s}=u_{s 1}+u_{S 2},
\end{gathered}
$$

where $u_{a}$ is adjustable feedforward controller used to compensate the model by parameter adaption, $u_{s}$ is the robust controller in which $u_{s 1}$ is linear feedback to stabilize the system, and $u_{s 2}$ is used to attenuate the parameter estimation error and nonlinear term of the model. In (57), the vector $\theta_{1}$ are all unknown parameters that need to be estimated. Therefore, the vector $\theta_{1}$ will be replaced by the $\widehat{\theta}_{1}$, the estimated value of the vector $\theta_{1}$, in the controller. Designing $u$ as $V_{2}=(L / 2) e_{2}^{2}+(1 / 2) \tilde{\theta}_{1}^{\mathrm{T}} \widetilde{\theta}_{1}$, then

$$
\dot{V}_{2}=L e_{2} \dot{e}_{2}-\tilde{\theta}_{1}^{\mathrm{T}} \dot{\hat{\theta}}_{1}=e_{2}\left(u-\mathbf{W}_{1} \boldsymbol{\theta}_{1}\right)-\tilde{\theta}_{1}^{\mathrm{T}} \dot{\hat{\theta}}_{1} .
$$

Since the $u_{a}$ is adjustable feedforward controller used to compensate the model by parameter adaption, the $u_{a}$ is

$$
u_{a}=\mathbf{W}_{1} \widehat{\theta}_{1} \text {. }
$$

Letting $u_{s 1}=-K_{1} e_{2}$, we get

$$
\dot{V}_{2}=e_{2}\left(-K_{1} e_{2}+\mathbf{W}_{1} \hat{\theta}_{1}-\mathbf{W}_{1} \boldsymbol{\theta}_{1}\right)-\tilde{\theta}_{1}^{\mathrm{T}} \dot{\hat{\theta}}_{1}
$$

where $K_{1}$ is a positive feedback gain. In order to ensure the stability of the system, the derivative of $V_{2}$ should be less or equal to zero by letting

$$
-e_{2} \mathbf{W}_{1} \tilde{\theta}-\tilde{\theta}_{1}^{\mathbf{T}} \dot{\hat{\theta}}_{1}=0 .
$$

Solving equation (62) gives

$$
\dot{\hat{\theta}}_{1}=-\mathbf{W}_{1}^{\mathrm{T}} e_{2} \text {. }
$$

Therefore, the adaptation mechanisms for adjusting parameters are

$$
\begin{aligned}
& \dot{\hat{\theta}}_{11}=-I e_{2}, \\
& \dot{\hat{\theta}}_{12}=-\omega e_{2}, \\
& \dot{\hat{\theta}}_{13}=-\dot{I}_{d} Z .
\end{aligned}
$$

Substituting (60) into (57) and adding the $u_{s 2}$ give

$$
L \dot{e}_{2}=-K_{1} e_{2}+\mathbf{W}_{1} \tilde{\theta}_{1}+u_{s 2} \text {. }
$$


For the deterministic robust control, the robust term $u_{s 2}$ should be any continuous function which satisfies the following conditions:

$$
\begin{aligned}
& \text { (1) } e_{2}\left(\mathbf{W}_{1} \tilde{\theta}_{1}+u_{s 2}\right) \leq \varepsilon_{1}, \\
& \text { (2) } e_{2} u_{S 2} \geq 0,
\end{aligned}
$$

where $\varepsilon_{1}$ is a positive design parameter that can be set arbitrarily small. Furthermore, the Lyapunov function can be defined as follows:

$$
V_{s 1}=\frac{L}{2} e_{2}^{2}
$$

The derivative of the $V_{s 1}$ becomes

$$
\dot{V}_{s 1}=L e_{2} \dot{e}_{2} \text {, }
$$

Substituting (65) into (68) gives

$$
\dot{V}_{s 1}=-K_{1} e_{2}^{2}+e_{2}\left(\mathbf{W}_{1} \tilde{\theta}_{1}+u_{s 2}\right) \text {. }
$$

One example of $u_{s 2}$ satisfying the (66) is

$$
u_{s 2}(t)=-e_{2} \frac{\left(\mathbf{W}_{1} \boldsymbol{\theta}_{1 m}\right)^{2}}{\varepsilon_{1}}
$$

where $\boldsymbol{\theta}_{\mathbf{1} m}=\boldsymbol{\theta}_{\mathbf{1} \max }-\boldsymbol{\theta}_{\mathbf{1} \text { min }}, \boldsymbol{\theta}_{\mathbf{1} \text { max }}$ is the upper limit of the $\boldsymbol{\theta}_{\mathbf{1}}$, and $\boldsymbol{\theta}_{\mathbf{1 m i n}}$ is the lower limit of the $\boldsymbol{\theta}_{\mathbf{1}}$. Substituting (70) into (69) gives

$$
\dot{V}_{s 1}=-K_{1} e_{2}^{2}+e_{2}\left(\mathbf{W}_{1} \tilde{\theta}_{1}-e_{2} \frac{\left(\mathbf{W}_{1} \boldsymbol{\theta}_{1 m}\right)^{2}}{\varepsilon_{1}}\right) .
$$

From the definition of the $\boldsymbol{\theta}_{\mathbf{1} \boldsymbol{m}}$, the derivative of the $V_{s 1}$ satisfies

$$
\dot{V}_{s 1} \leq-K_{1} e_{2}^{2}+\left|e_{2}\right|\left|\mathbf{W}_{1} \boldsymbol{\theta}_{1 m}\right|\left(1-\left|e_{2}\right| \frac{\left|\mathbf{W}_{1} \boldsymbol{\theta}_{1 m}\right|}{\varepsilon_{1}}\right) .
$$

Case 1: if $\left|e_{2}\right|\left|\mathbf{W}_{1} \theta_{1 m}\right|>\varepsilon_{1}$, then

$$
\begin{aligned}
\left|e_{2}\right| \frac{\left|\mathbf{W}_{1} \boldsymbol{\theta}_{1 m}\right|}{\varepsilon_{1}}>1 & \longrightarrow\left(1-\left|e_{2}\right| \frac{\left|\mathbf{W}_{1} \boldsymbol{\theta}_{1 m}\right|}{\varepsilon_{1}}\right)<0 \\
& \longrightarrow\left|e_{2}\right|\left|\mathbf{W} \boldsymbol{\theta}_{m}\right|\left(1-\left|e_{2}\right| \frac{\left|\mathbf{W}_{1} \boldsymbol{\theta}_{1 m}\right|}{\varepsilon_{1}}\right)<0 \\
& \Rightarrow \dot{V}_{s 1} \leq-K_{1} e_{2}^{2} .
\end{aligned}
$$

Case 2: if $\left|e_{2}\right|\left|\mathbf{W}_{1} \theta_{1 m}\right|<\varepsilon_{1}$, then

$$
\begin{aligned}
\left|e_{2}\right| \frac{\left|\mathbf{W}_{1} \boldsymbol{\theta}_{1 m}\right|}{\varepsilon_{1}}>1 & \longrightarrow\left(1-\left|e_{2}\right| \frac{\left|\mathbf{W}_{1} \boldsymbol{\theta}_{1 m}\right|}{\varepsilon_{1}}\right)<0 \\
& \longrightarrow\left|e_{2}\right|\left|\mathbf{W} \boldsymbol{\theta}_{m}\right|\left(1-\left|e_{2}\right| \frac{\left|\mathbf{W}_{1} \boldsymbol{\theta}_{1 m}\right|}{\varepsilon_{1}}\right)<0 \\
& \Rightarrow \dot{V}_{s 1} \leq-K_{1} e_{2}^{2} .
\end{aligned}
$$

In the first case, the derivative of the $V_{s 1}$ satisfies

$$
\dot{V}_{s 1} \leq-K_{1} e_{2}^{2} \leq-2 \frac{K_{1}}{L} V_{s 1} \text {. }
$$

The following inequality then can be derived by integrating (75). That is,

$$
V_{s 1}(t) \leq \exp \left(\frac{-2 K_{1} t}{\|L\|_{\max }}\right) V_{s 1}(0),
$$

where $t$ is time variant and $\|L\|_{\max }$ is the upper bound of $L$. According to Bellman-Gronwall, the positive definite $V_{s 1}$ is bounded by

$$
V_{s 1}(t) \leq \exp \left(\frac{-2 K_{1} t}{\|L\|_{\max }}\right) V_{s}(0)
$$

Therefore, $e_{2}$ is bounded by

$$
e_{2}(t) \leq \exp \left(-K_{1} t\right) \cdot e_{2}(0) \text {. }
$$

In the second case, the derivative of the $V_{s 1}$ satisfies

$$
\dot{V}_{s 1} \leq-K_{1} e_{2}^{2}+\varepsilon_{1} \leq-2 \frac{K_{1}}{L} V_{s 1}+\varepsilon_{1} \text {. }
$$

The following inequality then can be derived by integrating (79). That is,

$$
V_{s 1}(t) \leq \exp \left(\frac{-2 K_{1} t}{\|L\|_{\max }}\right) V_{s 1}(0)+\int_{0}^{t} \exp \left(\frac{-2 K_{1}(t-v)}{\|L\|_{\max }}\right) \varepsilon_{1} \mathrm{~d} v,
$$

where $t$ is time variant and $\|L\|_{\max }$ is the upper bound of $L$. According to Bellman-Gronwall, the positive definite $V_{s 1}$ is bounded by

$$
V_{s 1}(t) \leq \exp \left(\frac{-2 K_{1} t}{\|L\|_{\max }}\right) V_{s}(0)+\frac{\varepsilon\|L\|_{\max }}{2 K_{1}}\left[1-\exp \left(\frac{-2 K_{1} t}{\|L\|_{\max }}\right)\right] .
$$

Therefore, $e_{2}$ is bounded by

$$
e_{2}(t) \leq \exp \left(-K_{1} t\right) \cdot e_{2}(0)+\frac{1}{K_{1}}\left[1-\exp \left(-K_{1} t\right)\right]
$$

From the above inequalities, the tracking error of the twisted-string system exponentially approaches to a ball with converging rate $K_{1}$. Within a finite duration, the asymptotic output tracking is achieved. Furthermore, the control input $u$ can be derived as follows:

$$
u=-K_{1} e_{2}+W_{1} \widehat{\theta}_{1}+u_{s 2}
$$

In this section, we present simulation studies to illustrate the performance of the adaptive robust controller based on the force. The following parameters are used: the mass $m$ of the load is $0.5 \mathrm{~kg}$, the stiffness of the spring generating the external force is $4000 \mathrm{~N} / \mathrm{m}$, the armature resistant of $R$ of the DC motor is $9.7 \mathrm{ohm}$, the inductance of the DC motor is $0.0003 \mathrm{H}$, the torque constant $K t$ of DC motor is 0.1186 , and the back EMF constant $K b$ of DC motor is 0.0611 . The controller parameters are designed by selecting $k_{1}=10$, 
TABle 3: Parameters of the ARC controller.

\begin{tabular}{lcc}
\hline Parameter & Lower bound & Higher bound \\
\hline$\Theta_{1}$ & 0.001 & 0.0015 \\
$\Theta_{2}$ & 0.004465 & 0.006465 \\
$\Theta_{3}$ & 0 & 3.788 \\
$\Theta_{4}$ & 0 & 10.93 \\
$R$ & 8 & 12 \\
$k_{b}$ & 0 & 0.5 \\
$L$ & 0 & 0.0003 \\
\hline
\end{tabular}

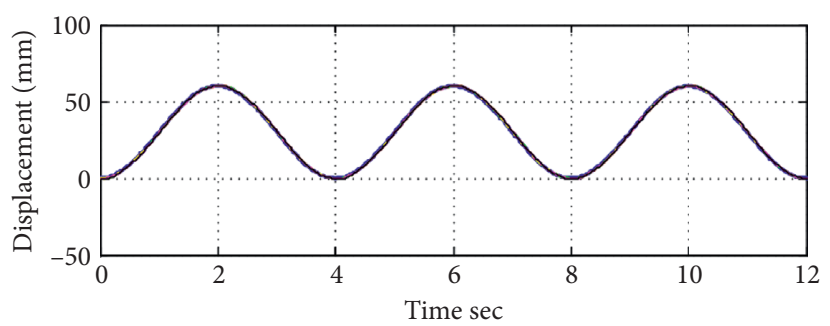

(a)

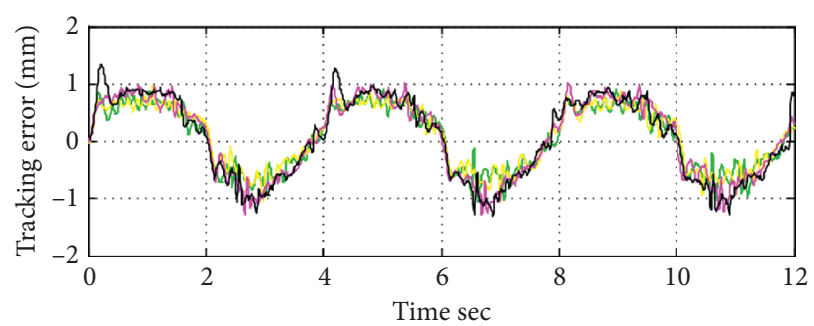

(b)

FIgURE 18: Experimental outcome of the ARC controller (sinusoidal motion).

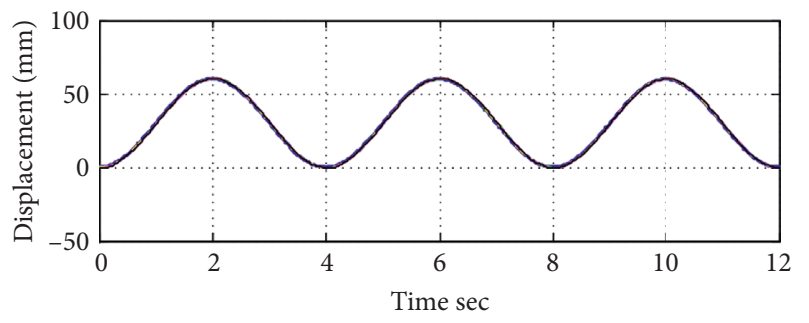

(a)

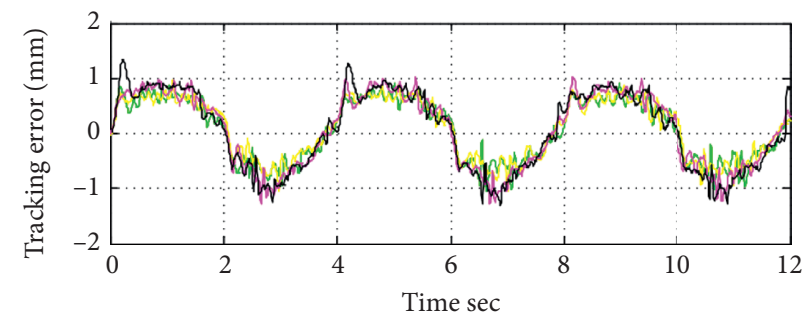

(b)

FIGURE 19: Experimental outcome of the ARC controller (trapezoidal motion).

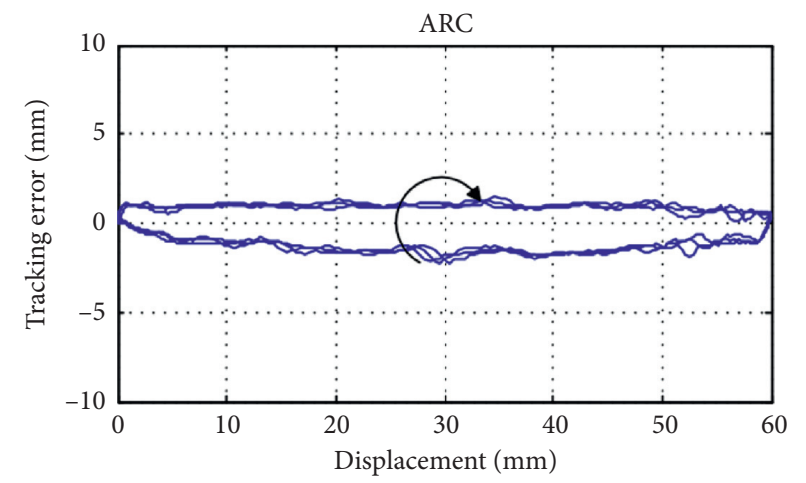

(a)

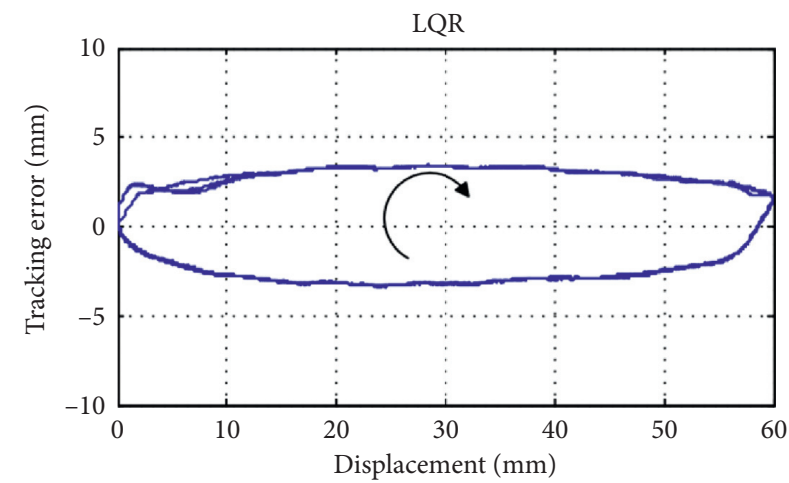

(b)

FIgURE 20: Comparison of tracking errors of ARC and LQR controllers (sinusoidal motion). 


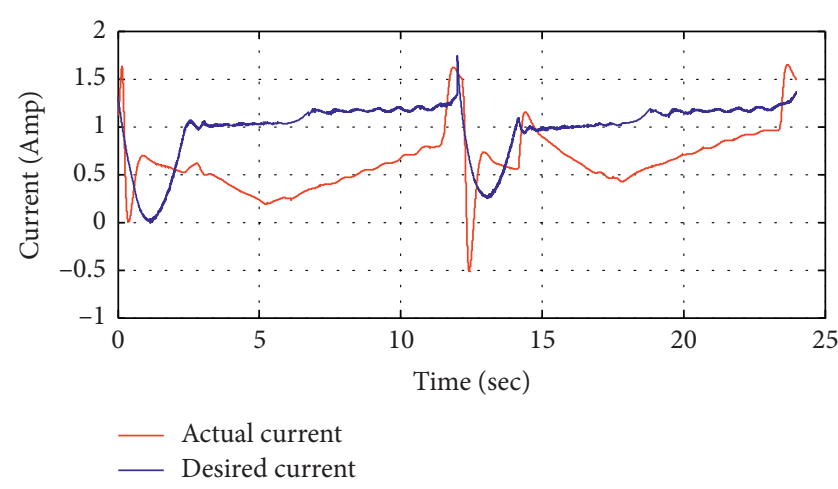

FIgURE 21: Comparison between actual current and desired current (sinusoidal motion).

$K=3$, and $K_{1}=7$. According to the system properties, the mass $m$ of the load is $0.5 \mathrm{~kg}$, the stiffness of the spring generating the external force is $4000 \mathrm{~N} / \mathrm{m}$, and the bounded unknown parameters are specified in Table 3.

\section{Conclusion}

In this study, we have proposed a method to estimate the contracted/expanded length of twisted-string actuation. In this study, two types of control structures will be investigated to reduce the effect of the unknown disturbances and the nonlinearity of the twisted-string actuation. They are (1) LQR controller with the force feedback and (2) ARC controller with the force feedback. The goal of the controller is to compensate for the tracking performances of the twistedstring actuators. Figures 18 and 19 demonstrate the experimental results of tracking performance of the ARC controller for both sinusoidal and trapezoidal motions. For such a sinusoidal motion, the amplitude of the periodic displacement is $60 \mathrm{~mm}$ and the duration of each cycle is 12 seconds. The ARC control scheme with force feedback demonstrates excellent results for tracking performance. It is clear that the ARC controller can improve both the transient and steadystate tracking performance over that of the LQR controller from Figure 20. From both Figures 18 and 19, the maximal tracking error of the proposed controller is $\sim 2 \mathrm{~mm}$. It is clear that either tracking errors of desired trajectory or the difference between the desired virtual input and actual virtual input (armature current of the DC motor) asymptotically approaches to zero, which proves the feasibility of the proposed controller, as shown in Figure 21. Nonlinearity and unknown external disturbance were big issues of twistedstring actuation, which can make the controller design much more difficult. With the proposed methods, the twisted string can demonstrate effective controlled outcome. However, some other methods still need to be developed. An eventtrigger based adaptive control scheme should be developed for the twisted-string actuation systems with unknown external disturbance. Under the event-trigger based adaptive control scheme [10], the effects caused by external disturbance and unknown modeling error can be compensated by constructing the estimator of its unknown constant upper bound.

\section{Data Availability}

The data used to support the findings of this study were supplied by the Nature Science Funding of China under rules and so cannot be made freely available. Requests for access to these data should be made to Lei Jiang, leijiang@ cumtb.edu.cn].

\section{Conflicts of Interest}

The author declares that they have no conflicts of interest.

\section{References}

[1] L. Jiang, R. Shisheie, M. Cheng, and E. Bakhoum, "Controller synthesis for assistive robotic device using twisted-string actuation," in Proceedings of the 2015 American Control Conference (ACC), pp. 2248-2253, Chicago, IL, USA, July 2015.

[2] G. Palli, C. Melchiorri, G. Vassura et al., "The DEXMART hand: mechatronic design and experimental evaluation of synergy-based control for human-like grasping," The International Journal of Robotics Research, vol. 33, no. 5, pp. 799-824, 2014.

[3] T. Sonoda and I. Godler, "Multi-fingered robotic hand employing strings transmission named "Twist Drive"” in Proceedings of the 2010 IEEE/RSJ International Conference on Intelligent Robots and Systems (IROS), pp. 2733-2738, CHN, Taipei, Taiwan, October 2010.

[4] M. H. Cheng, L. Jiang, Q. Mou et al., "Design, fabrication, and control of twisted actuated robotic device," in Proceedings of the 2016 American Control Conference, pp. 1215-1220, Boston, MA, USA, July 2016.

[5] M. Suzuki, T. Mayaha, and A. Ishizake, "Redundant muscle coordination of a multi-DOF robot joint by online optimization," in Proceedings of International Conference of AIM, pp. 1-6, Zurich, Switzerland, September 2007.

[6] T. Würtz, C. May, H. Benedikt, C. Natale, G. Palli, and C. Melchiorri, "The twisted string actuation system: modeling and control," in Proceedings of International Conference of AIM, pp. 1215-1220, Montreal, Canada, July 2010.

[7] G. Palli, C. Natale, C. May, C. Melchiorri, and T. Wurtz, "Modeling and control of the twisted string actuation system," IEEE/ASME Trans on Mechatronics, vol. 18, no. 2, pp. 664673, 2012.

[8] I. Gaponov, D. Popov, and J.-H. Ryu, "Twisted string actuation systems: a study of the mathematical model and a comparison of twisted strings," IEEE/ASME Transactions on Mechatronics, vol. 19, no. 4, pp. 1331-1342, 2014.

[9] R. Shisheie, L. Jiang, L. Banta, and M. Cheng, "Modeling and control of a bidirectional twisted-string actuation for an upper arm robotic device," in Proceedings of the 2015 American Control Conference, pp. 5794-5799, Chicago, IL, USA, July 2015.

[10] J. Cai, R. Yu, B. Wang, C. Mei, and L. Shen, "Decentralized event-triggered control for interconnected systems with unknown disturbances," Journal of the Franklin Institute, vol. 357, no. 3, pp. 1494-1515, 2020. 